\title{
Les carrières salariales des hommes et des femmes : quelle convergence sur longue période?
}

Wage careers of men and women: the assumption of a convergence

Fabienne Berton, Jean-Pierre Huiban et Frédérique Nortier

\section{OpenEdition}

Journals

Édition électronique

URL : http://journals.openedition.org/travailemploi/5048

DOI : $10.4000 /$ travailemploi.5048

ISSN : 1775-416X

Éditeur

DARES - Ministère du Travail

Édition imprimée

Date de publication : 15 mars 2011

Pagination : 9-25

ISSN : 0224-4365

Référence électronique

Fabienne Berton, Jean-Pierre Huiban et Frédérique Nortier, «Les carrières salariales des hommes et des femmes : quelle convergence sur longue période? », Travail et Emploi [En ligne], 125 I janvier-mars 2011, mis en ligne le 24 janvier 2012, consulté le 01 mai 2019. URL : http://journals.openedition.org/ travailemploi/5048 ; DOI : 10.4000/travailemploi.5048 


\title{
Les carrières salariales des hommes et des femmes : quelle convergence sur longue période?
}

\author{
Fabienne Berton (*), Jean-Pierre Huiban (**) et Frédérique Nortier (***)
}

\begin{abstract}
Peut-on soutenir l'hypothèse d'une convergence des carrières salariales entre hommes et femmes sur le long terme en France et dans quel sens? Telle est la question à laquelle répond cet article. Les carrières salariales des salariés français du secteur privé sont analysées à partir des données de l'Échantillon inter-régimes de cotisants 2001 (EIC 2001). Deux générations sont comparées, celles des individus nés en 1946 et en 1962 à l'aide d'une méthode qui articule analyse typologique et estimation de fonctions de gain. Dans le cas de la génération la plus ancienne, les hommes et les femmes présentent des profils de carrière salariale très différents. Puis, entre la première et la seconde génération, les trajectoires salariales des hommes et des femmes convergent vers un modèle différent du modèle masculin précédent. Les carrières sont fréquemment longues, mais entrecoupées de nombreuses interruptions, dont la fréquence augmente chez les femmes mais également chez les hommes. Les inégalités salariales entre hommes et femmes persistent, mais prennent des formes nouvelles, coexistant avec la montée d'une hétérogénéité interne à chacune des deux populations.
\end{abstract}

Depuis trente ans les carrières professionnelles se sont profondément transformées. L'apparition du chômage de masse et des situations de précarité, le développement de la flexibilité du temps de travail, l'instabilité du contexte conjoncturel, la modification des comportements féminins sur le marché du travail ont conduit à une remise en cause de ce qui est parfois présenté comme une forme canonique de carrière salariale (notamment masculine) : ascendante et sans interruption(s) significative(s) des débuts dans la vie active au départ en retraite.

L'objectif de ce travail, qui réunit à la fois chercheurs et responsables d'études dans les régimes de retraite complémentaire français ( $c f$. BERTON, Huiban, Nortier, 2009), est de fournir une analyse fine de ces carrières professionnelles dans le secteur privé, y compris à travers leurs interruptions et leurs discontinuités, afin d'évaluer ultérieurement les impacts des modifications réglementaires et économiques sur les montants de retraite individuelle. Son originalité tient d'abord aux données mobilisées. En général, les données utilisées pour l'estimation des salaires (les $D A D S(1)$ ) ne fournissent pas d'indications sur les périodes autres que celles relatives à la perception d'un salaire dans le secteur privé. La base mobilisée ici, l'EIC 2001 (pour Échantillon

(*) LISE CNRS, CNAM; fabienne.berton@cnam.fr (**) INRA, UR 1303 ALISS Ivry et ERUDITE-TEPP, université Paris-Est-Créteil ; jean-pierre.huiban@ivry.inra.fr (***) AGIRC-ARRCO; fnortier@agirc-arrco.fr

(1) Déclarations annuelles de données sociales.
Inter Régimes de Cotisants; cf. encadré 1) est un fichier d'origine administrative mis en place par la DREES(2) pour évaluer les droits acquis dans les différents régimes de retraite : l'évènement premier qui motive l'inscription à ce fichier (Colin, EL Mekradi de Freitas, Michaudon, 2004) est la cotisation à un régime obligatoire de retraite. Ce panel d'individus permet d'améliorer la connaissance des carrières en ajoutant aux salaires les périodes hors de l'emploi dans la mesure où ces périodes ouvrent des droits à la retraite (chômage indemnisé, maladie, etc.).

Notre travail propose aussi un choix méthodologique original ( $c f$. encadrés 2 et 3 ). En effet, un examen de la littérature sur les carrières individuelles révèle la coexistence de deux types d'approche. $\mathrm{La}$ première consiste en l'estimation économétrique de fonctions de gain (MINCER, 1974), soit la relation qui lie le salaire individuel à l'éducation et à l'expérience professionnelle, éventuellement complétée par d'autres variables explicatives (comme le sexe, le secteur d'activité ou la taille de l'entreprise, etc.). D'autres travaux ( $c f$. par exemple BRIARD, 2007) utilisent les différentes techniques de l'analyse des données pour proposer des typologies de carrières salariales, en analysant soigneusement les conséquences des accidents (comme le chômage ou la maladie) dans la forme de la carrière. Les deux

(2) DREES, Direction de la Recherche, des Études, de l'Évaluation et de la Santé du ministère du Travail, de l'Emploi et de la Santé (secteur Santé). 
approches sont complémentaires. L'utilisation de typologies permet de mieux caractériser l'hétérogénéité des carrières individuelles en mettant davantage l'accent sur les phénomènes de continuité et de rupture que sur les caractéristiques observables des individus. De façon symétrique, l'estimation d'une fonction de gain propre à chacune des souspopulations constitue un élément supplémentaire de caractérisation du type de carrière concerné.

C'est dans cet esprit que se structure cet article. Une première partie introduit notre hypothèse centrale, celle d'une convergence de la forme des carrières salariales entre hommes et femmes principalement initiée par une rupture de tendance au cours des années 1975-1985. Une deuxième partie présente les données que nous exploitons. La troisième partie expose nos résultats, qui s'appuient sur l'articulation des deux méthodes évoquées plus haut: une estimation des fonctions de gain sur l'ensemble de la population précède l'analyse typologique; l'examen des classes obtenues est ensuite complété par les résultats de l'estimation d'une fonction de gain propre à chaque sous-population concernée. La conclusion rappelle le principal résultat : la convergence entre les carrières masculines et féminines selon un modèle original, différent du modèle canonique des carrières masculines de l'après-guerre.

\section{L'hypothèse de convergence des carrières salariales entre hommes et femmes}

Notre sujet se situe au carrefour de trois ensembles de travaux : ceux sur les déterminants des salaires, notamment masculins (MAGNAC, Roux, 2009), ceux sur les transformations de la mobilité professionnelle (DE LARQUier, Remillon, 2008; Amossé, Ben Halima, 2010) où le salaire n'est pas la variable principalement mobilisée et ceux sur les comparaisons des salaires hommes / femmes (MEURS, Ponthieux, 2006) qui généralement ne développent pas d'analyse en termes de forme de carrière, mais en termes d'inégalité ou de discrimination.

Si la carrière salariale désigne au sens strict la mobilité d'un individu à l'intérieur de la hiérarchie des salaires (Lollivier, PAYEN, 1990), une telle acception ne suffit pas à rendre compte de notre problématique. Nous considérons que la carrière est formée d'une succession d'états, de l'inactivité à l'emploi salarié. La carrière salariale se compose non seulement de la succession d'épisodes plus ou moins longs d'emploi dans une ou plusieurs entreprises et de leur contrepartie salariale, mais aussi de différentes phases d'interruption ou d'accident de parcours. De très nombreux travaux sont consacrés aux déterminants du salaire individuel et à son évolution ( $c f$. les recensions de WILLIS, 1986 ou de CARD, 1999). Mais l'analyse du salaire et de ses déterminants n'est qu'une composante de la question traitée. Cette composante est d'autant plus importante que l'état d'activité salariée prédomine dans la carrière. Or, la tendance est à une fréquence plus grande de la non-activité au sens salarial, quelle qu'en soit la raison : chômage, maladie, retrait momentané ou non, volontaire ou involontaire du marché du travail, changement de statut professionnel, etc. L'analyse de ces ruptures prend donc toute son importance à côté des analyses plus traditionnelles des salaires, dès lors qu'elles ne peuvent plus être considérées comme exceptionnelles mais comme des composantes normales ou du moins fréquentes des carrières.

Pour aborder un objet aussi complexe qu'une carrière, la distinction entre hommes et femmes constitue une bonne grille de lecture. Elle permet d'abord de comprendre et décrire ce qui fonde les carrières salariales d'une génération donnée. Elle constitue également un bon moyen de décrire les évolutions survenues en testant notamment l'hypothèse d'une convergence entre sexes au cours du temps. Dans cet esprit, Karine BRIARD (2007) montre comment, pour les générations de l'avant et du début du baby-boom, le modèle masculin de la carrière longue et continue du salarié du secteur privé s'oppose à celui des carrières féminines brèves où le retrait du marché du travail s'effectue précocement. Pour les générations plus jeunes, les parcours longs mais avec davantage de discontinuité voient leur poids se renforcer aussi bien pour les hommes que pour les femmes. Dans les générations entrées sur le marché du travail à partir des années 1975 , le parcours des femmes s'améliore et dans le même temps, celui des hommes se dégrade. Le ralentissement salarial du début des années 1980 est pleinement ressenti pour les hommes à partir des générations nées au début des années 1960. Leurs salaires de début de carrière n'augmentent plus par rapport à ceux de la génération précédente. Pour les femmes, en revanche, les salaires de début de carrière progressent, quelle que soit la génération (NORTIER, RASS, 2006).

Ces premiers travaux suggèrent donc une transformation de la forme des carrières des hommes et des femmes et en indiquent les directions possibles. L'ensemble s'appuie sur le constat d'un certain nombre d'évolutions du contexte socio-économique. De fait, entre les années 1960 et les années 1980, des transformations sont intervenues qui ont conduit à une rupture de la forme générale des carrières au cours des années 1975-1985:

- des transformations structurelles de la population active et des activités économiques : hausse des niveaux d'éducation, tertiarisation et féminisation des emplois;

- la désindexation des salaires sur les prix depuis 1982; le niveau élevé atteint par la proportion de salariés rémunérés au Smic (Demailly, LE Minez, 1999);

- l'apparition d'un chômage élevé et le développement du sous-emploi et des mobilités contraintes. 
Un premier effet de ces transformations est l'accroissement de l'instabilité professionnelle. Les salariés récents semblent devenus beaucoup plus substituables aux salariés anciens (GIVORD, MAURIN, 2003). On observe une montée générale de l'instabilité. Du côté de la demande de travail issue des entreprises, en 2000, selon Marie LeCLAIR et Sébastien Roux (2006), $19 \%$ des heures rémunérées étaient le fait de salariés restant moins d'un an dans leur entreprise. Les mêmes auteurs (LeCLAIR, Roux, 2007), notent la multiplication des emplois de courte durée (occupés moins d'un an) et suggèrent deux interprétations : la recherche de flexibilité et la volonté d'estimer en situation la productivité des salariés. Luc BeHAGHEL (2003) nuance ce constat en l'expliquant par un déclin des contrats de travail de long terme à l'embauche sans rupture des contrats existants : l'insécurité de l'emploi, mesurée par le taux de transition annuel de l'emploi vers le nonemploi a considérablement augmenté en France, mais elle se polarise sur certaines catégories de salariés, les peu qualifiés et ceux de faible ancienneté.
Un second effet de ces transformations touche la forme générale des carrières : un très grand nombre de travaux signale une rupture sensible au cours des années 1975-1985 (Debrand, Privat, 2002). Les écarts entre tranches d'âge se sont creusés, l'âge où la rémunération est maximale s'est élevé. Pour les générations 1930 à 1954, les salaires de début d'activité progressent fortement d'une génération à l'autre; pour les générations 1955 à 1975, qui arrivent sur le marché du travail à partir du milieu des années 1970, ces écarts se réduisent fortement, sauf pour les diplômés de l'enseignement supérieur (Buchinsky, FougÈre, Kramarz, 1998), si bien que les différences de carrière salariale entre des générations éloignées dans le temps ne peuvent pas s'expliquer uniquement par l'accumulation de capital humain. Au cours des décennies récentes, le rendement de l'éducation a décru, alors que la structure de qualification de la population active a augmenté. Toutefois les différentiels de salaire entre les différents niveaux de formation ou de diplôme tendent à se maintenir.

\section{Encadré 1 \\ Les données (EIC 2001) et la reconstitution des salaires}

Les données que nous mobilisons proviennent de l'Échantillon inter-régimes de cotisants 2001 (EIC 2001). Ce fichier, conçu afin d'évaluer les droits à pension acquis par les actifs dans les régimes de retraite obligatoires, constitue un panel de données individuelles. La DREES a collecté les rémunérations perçues, les droits acquis annuellement et les informations sur les périodes de chômage et de maladie auprès de l'ensemble des régimes obligatoires de retraite, de l'Unédic, de l'Insee, de la Comptabilité Publique et du Ministère de la Défense.

L'EIC est constitué d'individus nés en octobre des années paires afin de permettre l'appariement avec les données extraites du panel DADS. L'échantillon 2001 porte sur les individus âgés de 31 à 67 ans au 31 décembre 2001 (nés entre 1934 et 1970). Son taux de sondage est d'une génération sur quatre et de 2,67 \% d'individus par génération tirée. L'extrait que nous utilisons concerne les individus pour lesquels au moins une période cotisée à I'ARRCO (1) ou à l'AGIRC (2) a été enregistrée et qui ne sont pas encore partis à la retraite au 31 décembre 2001 (soit les générations de 1946 à 1970). L'échantillon contient 135000 individus et plus de 2 millions d'observations individu/année.

Une des difficultés rencontrées tient à la reconstitution du salaire des cadres. Nous disposons de deux sources : les $D A D S$ qui ne sont renseignées dans l'EIC qu'à partir de 1976 et les déclarations du régime de base de retraite, qui ne couvrent que la partie du salaire sous le plafond de la Sécurité sociale jusqu'en 1999. Or, en moyenne $14 \%$ des salariés, principalement des cadres, ont un salaire supérieur à ce plafond sur l'ensemble de la période étudiée. Nous avons estimé les salaires des cadres à partir des droits acquis annuellement au régime AGIRC. Le rapprochement des deux sources de données montre que les moyennes de salaire annuel brut ont un niveau et une évolution sensiblement équivalents quelle que soit la source. Au final, ce sont les salaires annuels bruts estimés à partir des données des régimes de retraite que nous conservons.

La durée du travail (hebdomadaire, mensuelle ou annuelle) est une variable inexploitable en l'état car renseignée de façon incomplète dans l'EIC. II nous est de ce fait impossible de distinguer entre le travail à temps complet, à temps partiel et les contrats à durée déterminée.

L'EIC ne contient pas de renseignement sur les diplômes et les niveaux de qualification. Nous avons calculé un âge effectif d'entrée dans la vie active en considérant qu'il correspondait à la première année avec quatre trimestres validés. L'âge moyen de début de carrière ainsi mesuré passe de 18 ans et demi pour la génération 1946 à pratiquement 22 ans pour la génération 1970, reflétant l'allongement à la fois des études et de l'insertion professionnelle.

(1) Association du régime de retraite complémentaire des salariés.

(2) Association générale des institutions de retraite des cadres. 


\section{La sélection de deux générations et la caractérisation des parcours professionnels}

Les données exploitées (EIC 2001) sont structurées par génération ( $c f$. encadré 1). Les générations pour lesquelles nous disposons de suffisamment d'informations s'échelonnent de 1946 à 1970 (dates de naissance des individus dont on étudie la carrière).

\section{Le choix de deux générations : 1946 et 1962}

Nous retenons deux générations. La génération des individus nés en 1946 est la plus ancienne pour laquelle nous disposons de données. Elle débute son activité professionnelle dans l'environnement socio-économique des années 1960, bien avant la rupture de tendance signalée dans la littérature présentée plus haut et, à cet égard, elle est représentative des carrières des générations du début du baby-boom. De façon symétrique, la génération 1962 est la dernière génération pour laquelle nous disposons de suffisamment d'années d'expérience professionnelle observables (23 ans). C'est la première génération pour laquelle les ruptures de tendance évoquées feraient pleinement sentir leurs effets, puisqu'elle entre dans la vie active au début des années 1980. Afin de rendre les deux populations comparables, nous nous sommes limités aux individus âgés de 16 à 39 ans, seules catégories d'âge présentes dans les deux générations.

\section{Des parcours professionnels caractérisés par treize états}

Une première étape consiste à caractériser les parcours professionnels individuels. L'individu est observé chaque année et sa situation est définie par un état annuel exclusif. C'est la succession de ces états annuels qui constitue sa carrière. Pour bien définir ces états annuels, nous disposons en premier lieu de trois catégories d'information : le nombre de trimestres cotisés au titre d'un emploi salarié, le nombre de trimestres validés au titre d'une interruption de carrière de type chômage ou maladie et l'absence de trimestre cotisé ou validé. L'ensemble nous conduit à définir plusieurs types d'états :

- l'inactivité totale avant le début de l'activité ou en cours de carrière (aucun trimestre, ni cotisé ni validé); - 1'activité partielle (de un à quatre trimestres validés avec éventuellement un trimestre cotisé, c'est-à-dire du chômage ou de la maladie pour une partie ou la totalité de l'année avec éventuellement un trimestre d'emploi salarié et/ou d'inactivité);

- l'emploi en dehors du secteur privé (fonctionnaires, indépendants);

- l'emploi dans le secteur privé. Dans ce dernier cas, deux situations peuvent se présenter : soit l'activité salariée est continue dans l'année avec quatre trimestres cotisés, soit l'activité salariée est discontinue dans l'année sans qu'un autre état puisse être repéré avec de un à trois trimestres cotisés. Le salaire individuel annuel brut est dans ces deux cas classé en fonction de sa place dans la distribution des salaires annuels bruts de la génération étudiée, quelle que soit la durée effective de travail de l'individu.

Tableau 1 : Codification des treize états annuels individuels utilisés pour décrire les carrières (SITU)

\begin{tabular}{|c|c|}
\hline \multicolumn{2}{|c|}{ Avant l'activité professionnelle } \\
\hline $\mathrm{Y}$ & Avant le début d'activité professionnelle (études, inactivité ou insertion professionnelle) \\
\hline \multicolumn{2}{|c|}{ Emploi salarié seul } \\
\hline $\mathrm{N}$ & Emploi salarié seul avec un salaire annuel brut ne permettant pas de valider quatre trimestres dans l'année \\
\hline $\mathrm{P}$ & Emploi salarié seul avec un salaire annuel brut $<=20$ premiers centiles et quatre trimestres validés \\
\hline $\mathrm{D}$ & Emploi salarié seul avec un salaire annuel brut $>=21^{\mathrm{e}}$ centile et $<=45^{\mathrm{e}}$ centile et quatre trimestres validés \\
\hline $\mathrm{T}$ & Emploi salarié seul avec un salaire annuel brut $>=46^{\mathrm{e}}$ centile et $<=60^{\mathrm{e}}$ centile et quatre trimestres validés \\
\hline Q & Emploi salarié seul avec un salaire annuel brut $>=61^{\mathrm{e}}$ centile et $<=85^{\mathrm{e}}$ centile et quatre trimestres validés \\
\hline $\mathrm{C}$ & Emploi salarié seul avec un salaire annuel brut $>=86^{\mathrm{e}}$ centile et quatre trimestres validés \\
\hline \multicolumn{2}{|c|}{ Interruptions dans l'année } \\
\hline $\mathrm{E}$ & Emploi salarié avec chômage et/ou maladie dans l'année et quatre trimestres validés \\
\hline$\| \mathrm{M}$ & Chômage et/ou maladie toute l'année et quatre trimestres validés \\
\hline \multicolumn{2}{|c|}{ Activité en dehors du secteur privé } \\
\hline A & Emploi dans d'autres régimes que salarié et quatre trimestres validés (avec éventuellement chômage et/ou maladie) \\
\hline $\mathrm{F}$ & Autres situations d'emploi avec quatre trimestres cotisés, éventuellement dans plusieurs secteurs d'activité et avec \\
\hline \multicolumn{2}{|c|}{ éventuellement chômage et/ou maladie } \\
\hline \multicolumn{2}{|c|}{ Lien distendu à l'activité professionnelle ou absence d'activité } \\
\hline $\mid \mathrm{X}$ & Entre un et trois trimestres validés autre qu'en emploi salarié seul \\
\hline I & Aucun trimestre validé \\
\hline
\end{tabular}




\section{Encadré 2}

\section{Les méthodes, fonctions de gain et analyse typologique}

Cette étude s'appuie sur l'utilisation complémentaire de deux approches : l'estimation économétrique de fonctions de gain individuelles et l'élaboration de typologies de carrière à partir d'une méthode de classification.

\section{Les fonctions de gain}

L'estimation économétrique de fonctions de gain constitue un des outils classiques de l'économie du travail. La forme la plus simplement associable à la notion de carrière individuelle consiste à relier $w_{i t}$ le niveau du salaire obtenu par l'individu $i$ l'année $t$ à $E X P_{i t}$ l'expérience qu'il a acquise sur le marché du travail, $\varepsilon_{i t}$ représentant un terme d'erreur dont la spécification dépend de l'estimateur utilisé (cf. encadré 3 pour une présentation détaillée du mode de construction des variables) :

$$
\log w_{i t}=\beta_{0}+\beta_{1} E X P_{i t}+\beta_{2} E X P_{i t}^{2}+\varepsilon_{i t}
$$

Le recours à une forme quadratique permet d'envisager la possibilité d'un retournement : le salaire cesserait de croître à partir d'un seuil égal à $-\frac{\beta_{1}}{2 \beta_{2}}$ années. La forme précédente repose sur l'hypothèse implicite que seule l'expérience accumulée par l'individu détermine le niveau de son salaire. Une telle hypothèse n'est bien évidemment pas fondée (LollivieR, PAYEN, 1990). D'autres caractéristiques, observables (nombre d'années d'études, sexe, caractéristiques de l'emploi occupé, savoir-faire de l'individu, etc.) ou non observables, influent sur le niveau du salaire, qu'il s'agisse ou non de discrimination (sexe, appartenance communautaire, etc.). L'omission de ces caractéristiques, si elles sont corrélées avec certaines variables explicatives présentes dans la régression, conduit classiquement à l'obtention de coefficients biaisés dans le cas de la forme précédente.

Deux voies peuvent être empruntées afin de résoudre ce problème. La première consiste à introduire d'autres caractéristiques mesurables dans la fonction de gain, toujours appliquée à l'ensemble de la population :

$$
\log w_{i t}=\beta_{0}+\beta_{1} E X P_{i t}+\beta_{2} E X P_{i t}{ }^{2}+\sum_{k=3}^{K} \beta_{k} x_{k i t}+\varepsilon_{i t}
$$

De nombreuses formes "augmentées» de la fonction de gain existent dans la littérature (WILLIS, 1986; CARD, 1999). Notons que ces variables ne concernent que très rarement des événements survenus en cours de carrière, telles des ruptures d'activité. Cette approche ne se prête qu'imparfaitement à la prise en compte de telles discontinuités. L'effet de celles-ci sur le niveau de salaire n'est alors qu'imparfaitement contrôlé à travers l'inclusion d'effets individuels dans le modèle économétrique. L'exploitation de l'EIC nous permettra d'introduire des variables caractérisant explicitement les interruptions d'activité (des "accidents»), qu'elles soient annuelles ou observées à un moment donné de la carrière.

Une seconde façon de procéder consiste à tenter de réduire l'hétérogénéité non observée en conduisant des estimations de fonctions de gain sur des sous-populations présumées plus homogènes du point de vue de la question considérée ici, c'est-à-dire la forme de la carrière individuelle. Mais comment constituer de telles populations ? L'estimation de fonctions séparées pour les hommes et pour les femmes constitue une première réponse. Nous estimerons ainsi la forme suivante, dans le cas des femmes puis des hommes présents dans notre fichier:

$$
\log w_{i t}=\beta_{0}+\beta_{1} E X P_{i t}+\beta_{2} E X P_{i t}^{2}+\beta_{3} E D U_{i}+\beta_{4} C A D_{i t}+\beta_{5} A C C A N_{i t}+\beta_{6} A C C I N D I V_{i}+\varepsilon_{i t}
$$

Le salaire perçu par l'individu $i$ durant l'année $t$ dépend donc de son expérience, mais aussi d'autres variables : le statut (variable indicatrice $C A D$, distinguant cadre et non cadre), le nombre d'années d'études $E D U$, et deux variables indiquant la présence d'une interruption d'activité, soit l'année observée $\left(A C C A N_{i t}\right)$, soit à un moment quelconque de la carrière $\left(A C C I N D I V_{i}\right)$. L'estimation séparée permet de comparer la forme des courbes respectives et fournit une information plus riche que la simple insertion d'une indicatrice de sexe au sein d'une estimation unique.

Mais l'hétérogénéité interindividuelle ne se réduit pas aux seules caractéristiques observables ou modélisables, comme le sexe. Il existe certes des méthodes d'estimation qui permettent de contrôler les effets de cette hétérogénéité et nous en utiliserons certaines, comme l'inclusion d'un effet fixe individuel. Mais cette hétérogénéité entre individus peut évoluer dans le temps, du fait notamment d'interruptions présentes à un ou plusieurs moments de la carrière. L'utilisation d'une méthode d'analyse typologique, au sein de laquelle les changements de situation vont jouer un rôle important, complétera donc utilement l'approche économétrique.

\section{L'analyse typologique}

En construisant une typologie des carrières salariales, nous poursuivons deux buts : la description de la forme générale des carrières et de leur transformation d'une génération à l'autre et l'estimation des fonctions de gain sur des classes de salariés a priori plus homogènes.

Pour être suffisamment discriminante, une typologie des carrières doit répondre à plusieurs objectifs : elle doit les différencier selon leur forme générale, selon la succession et l'ordre des différents états possibles et selon le temps passé dans ces états. II s'agit d'analyser les parcours en classant les états successifs (GRELET, 2002). La trajectoire est à ce stade décrite dans son ensemble en effectuant une analyse des correspondances multiples (ACM) sur les états aux différents âges/années. Une classification est ensuite opérée sur les 150 premiers facteurs représentant plus de $80 \%$ de l'inertie totale du nuage. La méthode de classification retenue est celle recommandée pour de gros échantillons (NAKACHE, ConfaIs, 2005), elle consiste à enchaîner deux méthodes distinctes :

- deux classifications non hiérarchiques à l'aide des centres mobiles. Celles-ci sont croisées afin d'obtenir des "formes fortes", 350 classes sont ainsi obtenues;

- une classification hiérarchique ascendante (CAH) est ensuite réalisée sur ces formes fortes. Le critère de classement retenu est le critère de Ward, qui maximise l'inertie inter-classe et minimise la variance intra-classe.

Cette classification est effectuée successivement pour les générations 1946 et 1962. On constate qu'en majorité les classes se retrouvent d'une génération à l'autre. Seuls évoluent leur composition et leur poids relatif au sein de l'ensemble de la génération. Appliquée à chacune des deux générations, cette méthode produit une typologie en douze classes. 
La variable SITU ( $c f$. tableau 1) constitue notre nomenclature des états annuels exclusifs les uns des autres et résume les différents événements de la carrière de chaque individu.

\section{Le déroulement de carrière des deux générations}

\section{Les statistiques descriptives et les chronogrammes}

Les statistiques du tableau 2 décrivent les différences entre la situation des hommes et celle des femmes et la façon dont elles évoluent entre les deux générations. D'une génération à l'autre, la moyenne des salaires annuels bruts progresse, ainsi que la part des cadres et des femmes. Mais la part des salariés touchés par une interruption d'activité (maladie et/ou chômage) une année donnée ou durant leur carrière progresse aussi, ce qui conduit à la réduction de la durée de l'ancienneté dans les emplois.

Par rapport aux hommes, le salaire annuel brut moyen des femmes augmente aussi tout en se situant à un niveau inférieur, sa dispersion (écarttype) en revanche se réduit, à la différence de celle des hommes. Les femmes se caractérisent par une progression plus rapide du statut cadre entre les deux générations, une plus forte progression des interruptions d'activité dans une année ou dans la carrière (elles ne correspondent pas uniquement aux maternités) et par conséquent une ancienneté dans les emplois non seulement plus faible que celle des hommes mais aussi moins proche de la durée de l'expérience professionnelle.

\section{Encadré 3}

\section{La définition des variables}

La variable SITU caractérisant la situation d'un salarié une année donnée et utilisée pour les chronogrammes et la classification a été précédemment définie. Les autres variables utilisées dans les statistiques descriptives ou les estimations sont les suivantes :

- le salaire : salaire annuel brut moyen en euros de 2007;

- le statut : variable indicatrice $C A D$, cadre (1) et non cadre (0);

- le nombre estimé d'années d'études EDU : âge estimé d'entrée en activité (quatre trimestres validés) moins l'âge légal de la fin de la scolarité obligatoire (14 ans/16 ans);

- l'interruption d'activité l'année observée $\left(A C C A N_{i t}\right)$ : présence d'une interruption professionnelle de six mois durant l'année observée;

- l'interruption d'activité à un moment quelconque de la carrière $\left(A C C I N D I V_{i}\right)$ : présence d'une interruption professionnelle de six mois, quelle qu'en soit la raison, à un moment quelconque de la carrière (entre 16 et 39 ans);

- l'expérience $\left(E X P_{i t}\right)$ : durée maximale de présence sur le marché du travail de l'individu i, soit la différence entre l'année de premier emploi (quatre trimestres validés) et l'année observée;

- l'ancienneté $\left(A N C_{i t}\right)$ : ancienneté d'activité professionnelle effective de l'individu à la date donnée. Concrètement cette variable est obtenue en déduisant de la précédente (EXP) le nombre d'années durant lesquelles l'individu "disparaît" du fichier des salaires. Un individu présent de 1981 à 1985, puis de 1991 à 1995 cumulera, en 1995, 15 années d'expérience et 10 années d'ancienneté.

Tableau 2 : Caractéristiques descriptives des deux générations par sexe de 16 à 39 ans

\begin{tabular}{|c|c|c|c|c|c|c|c|}
\hline & $\begin{array}{c}\text { Salaire } \\
\text { annuel moyen } \\
\text { (écart-type) }\end{array}$ & $\begin{array}{c}\text { Accident } \\
\text { dans l'année } \\
\text { (\% de salariés) }\end{array}$ & \begin{tabular}{|c|}
$\begin{array}{c}\text { Accident } \\
\text { dans la } \\
\text { carrière } \\
\text { (\% de salariés })\end{array}$ \\
\end{tabular} & $\begin{array}{l}\text { Expérience } \\
\text { moyenne } \\
\text { accumulée à } \\
39 \text { ans } \\
\text { (en années) }\end{array}$ & $\begin{array}{l}\text { Ancienneté } \\
\text { moyenne } \\
\text { accumulée à } \\
39 \text { ans } \\
\text { (en années) }\end{array}$ & $\begin{array}{c}\text { Cadres } \\
(\% \text { de salariés })\end{array}$ & $\begin{array}{c}\text { Poids du } \\
\text { genre dans la } \\
\text { génération } \\
\text { (\% de salariés) }\end{array}$ \\
\hline \multicolumn{8}{|l|}{ Hommes } \\
\hline $\begin{array}{l}\text { Génération } \\
\mathbf{1 9 4 6} \\
\mathrm{N}=8187\end{array}$ & $\begin{array}{l}16890 \\
(11631)\end{array}$ & 1,4 & 83,5 & 20,0 & 19,4 & 10,5 & 54,2 \\
\hline $\begin{array}{l}\text { Génération } \\
\mathbf{1 9 6 2} \\
\mathrm{N}=10922\end{array}$ & $\begin{array}{c}20788 \\
(13404)\end{array}$ & 5,7 & 89,5 & 18,6 & 17,3 & 11,7 & 51,2 \\
\hline \multicolumn{8}{|l|}{ Femmes } \\
\hline $\begin{array}{l}\text { Génération } \\
\mathbf{1 9 4 6} \\
\mathrm{N}=6912\end{array}$ & $\begin{array}{c}10860 \\
(20645)\end{array}$ & 1,6 & 83,3 & 19,5 & 17,2 & 3,3 & 45,8 \\
\hline $\begin{array}{l}\text { Génération } \\
\mathbf{1 9 6 2} \\
\mathrm{N}=10391\end{array}$ & $\begin{array}{c}14160 \\
(11628)\end{array}$ & 6,6 & 91,4 & 18,4 & 16,4 & 6,1 & 48,8 \\
\hline
\end{tabular}

Source : DREES, EIC 2001 et calculs CNAM-INRA-AGIRC/ARRCO. 
Les treize états annuels individuels que nous avons définis ( $c f$. supra) nous permettent d'examiner l'évolution globale de chaque génération 1946 et 1962, toutes caractéristiques professionnelles confondues. Pour cela nous recourons à une présentation graphique spécifique : les chronogrammes qui offrent l'avantage de visualiser la distribution de la génération considérée à chaque date (qui correspond à un âge pour les individus de la génération) et qui montrent ensuite de date en date les transformations de cette distribution entre les différents états définis et matérialisés dans le chronogramme par différentes nuances ( $c f$. tableau 1). Le graphique 1 présente les chronogrammes des carrières de 14 à 39 ans des hommes et des femmes des deux générations 1946 et 1962, de façon à visualiser aux mêmes âges les situations et les évolutions.

Pour la génération 1946, les hommes et les femmes connaissent des déroulements de carrière très différents. On constate pour les hommes le pic inversé d'inactivité entre 19 et 20 ans correspondant au service militaire, que l'on ne retrouve plus pour la génération 1962 et pour les femmes, différents pics inversés entre 20 et 30 ans correspondants aux maternités que l'on ne retrouve plus non plus dans la génération 1962. Les carrières féminines de la génération 1946 par rapport à celles des hommes de la même génération sont davantage marquées par le passage à l'inactivité, les bas salaires et différentes formes intermédiaires entre inactivité et emploi.

Pour la génération née en 1962, une première remarque concerne l'allongement de la situation avant l'entrée dans un premier emploi, qui correspond à la fois à l'allongement de la durée des études et à celle de l'insertion professionnelle. Une seconde remarque porte sur l'accroissement aussi bien pour les hommes que pour les femmes des situations annuelles qui associent emploi salarié et chômage et/ou maladie. Une dernière remarque touche à la disparition relative de la différence hommes/ femmes. Le déroulement des carrières de la génération 1962 montre la convergence générale de forme entre les hommes et les femmes. Notamment, la part de l'inactivité baisse nettement chez les femmes pour se rapprocher de celle des hommes. On note cependant pour les femmes un poids légèrement plus important des bas salaires, des interruptions de carrière au cours d'une année et des formes intermédiaires entre inactivité et emploi. Cette visualisation nécessite d'être confirmée et approfondie par le calcul.

\section{Les fonctions de gain : expérience théorique et ancienneté réelle}

Le tableau 3 présente les résultats d'estimation des fonctions de gain par sexe, dans le cas des générations 1946 et 1962 . Les comportements d'activité concernent les années 1962 à 1985 pour la génération 1946 et les années 1978 à 2001 pour la génération
1962. Parmi les estimateurs disponibles (CORNwELLRUPERT, 1988), nous avons retenu l'estimateur Within, complété, afin de fournir une estimation des coefficients associés aux variables stables dans le temps, par l'estimateur des variables instrumentales (3) dans la version proposée par HAUSMAN et TAYLOR (1981). Des indicatrices annuelles, censées corriger les effets de l'hétérogénéité temporelle cette fois, ont été introduites, dont les coefficients n'ont pas été reportés. D'autres estimateurs, comme les moindres carrés quasi généralisés (effets aléatoires individuels) n'ont pas été retenus, du fait d'un rejet consécutif à l'emploi du test d'Hausman.

Dans le cas de la génération 1946, les signes et ordres de grandeur obtenus dans le cas des hommes sont cohérents avec ceux rencontrés dans la littérature, et plus généralement avec les prédictions du modèle théorique. Le coefficient de l'expérience est positif et celui de l'expérience au carré est négatif. Le coefficient associé au statut de cadre est positif, tandis que ceux associés aux interruptions de carrière sont négatifs. Le rendement de l'éducation est significativement positif.

La comparaison entre la carrière des femmes et celle des hommes met en lumière des différences significatives. La progression annuelle du salaire des hommes est nettement plus rapide. L'accession au statut de cadre entraîne un effet positif plus affirmé pour les femmes. Dans le cas des interruptions de carrière, l'effet instantané (l'année où survient l'interruption) est plus fort chez les hommes mais l'effet permanent (sur l'ensemble de la carrière) est plus élevé chez les femmes. Enfin le rendement de l'éducation est l'occasion d'une dernière et très nette différenciation : il est positif chez les hommes et négatif chez les femmes. Rappelons cependant que cette variable est mesurée de façon très imparfaite et assimile une entrée tardive sur le marché du travail à la poursuite d'études plus longues. L'erreur de mesure peut donc être considérable et, plus grave encore, peut varier selon le sexe, d'où la prudence nécessaire dans l'interprétation de ce dernier résultat.

L'examen des résultats de la génération 1962 montre que, globalement, les différences observées entre les carrières des hommes et celles des femmes s'atténuent, pour tendre vers un modèle assez sensiblement différent du modèle des carrières masculines de la génération 1946. Les différents coefficients associés à la variable «expérience» ont tous très sensiblement diminué. La progression annuelle est beaucoup plus faible pour les hommes comme pour les femmes : le coefficient des hommes de la génération 1962 est désormais plus faible que celui des femmes de la génération 1946. L'impact

(3) Le lecteur non familiarisé avec ces aspects pourra se référer à BeHAGHEL (2006). 
du statut cadre est resté à peu près constant chez les hommes et a baissé chez les femmes. Le rendement moyen de l'éducation diminue très fortement chez les hommes et devient non significatif chez les femmes. Les effets des interruptions de carrière évoluent : les effets négatifs instantanés s'atténuent tandis que les effets permanents demeurent négatifs, notamment dans le cas des femmes.

L'ensemble de ces résultats suggère que les modèles de carrière individuelle, telle que les décrivent les fonctions de gain, évoluent dans un sens qui n'est plus le modèle masculin de la génération 1946, marqué notamment par un rendement élevé de l'expérience. Plus généralement, la bonne qualité initiale de l'ajustement du modèle se dégrade dans le cas des femmes de la génération 1946 et dans celui de la génération 1962 aussi bien pour les hommes que pour les femmes. Le modèle théorique sous-jacent à cette première estimation rend bien compte de la réalité masculine de la génération 1946, il n'est en revanche adapté ni à la situation féminine de la génération 1946, ni aux situations masculine et féminine de la génération 1962. Ceci nous conduit à en amender la forme. Nous allons désormais considérer que ce n'est plus l'expérience telle que la définissait la date d'entrée sur le marché du travail, mais l'ancienneté effective en situation d'emploi qui compte. En procédant à cette substitution et en laissant inchangé le reste, nous obtenons des résultats différents, plus lisibles et plus convaincants ( $c f$. tableau 4). Les coefficients associés à cette nouvelle variable diffèrent de ceux associés à l'ancienne, mais les coefficients associés aux autres variables explicatives sont également modifiés.

Dans le cas des hommes de la génération 1946, les valeurs obtenues pour les différents coefficients varient peu, sauf dans le cas de la variable «interruption de carrière», dont l'impact négatif devient un peu plus faible : visiblement une partie de cet effet a été intégrée dans l'impact du changement de variable. Dans le cas des femmes, l'ensemble des coefficients est très nettement modifié. Le coefficient de la variable «ancienneté» fait plus que doubler par rapport à celui obtenu précédemment pour la variable «expérience». Sa valeur dépasse celle obtenue dans le cas des carrières masculines. La carrière des femmes évolue donc beaucoup plus vite lorsqu'on utilise cet indicateur. De façon plus surprenante, le rendement de la variable «éducation» s'accroît lui aussi très significativement pour dépasser celui des hommes. L'examen des résultats relatifs à la génération 1962 confirme nettement l'impact de ce changement de variable. Le rendement d'une année d'ancienneté ne décroît que faiblement entre 1946 et 1962 dans le cas des carrières masculines et il augmente dans celui des carrières féminines. C'est aussi le cas du rendement de l'éducation.

L'ensemble des résultats issus de cette comparaison suggère une interprétation d'ensemble. Ce qui différencie fondamentalement la carrière des hommes de celles des femmes, et la carrière des salariés de la génération 1946 de celle des salariés de la génération 1962, n'est pas tant la dynamique observée dans le cas des carrières continues que l'accroissement du poids des discontinuités au sein des carrières. Les hommes de la génération 1946 ont accumulé en moyenne 20,07 années d'expérience à 39 ans, dont 19,42 années d'activité effective. La situation des femmes diffère dès cette génération : 19,47 années d'expérience pour 17,24 années d'activité effective. Pour la génération 1962, toutes ces valeurs sont à la baisse mais surtout l'écart entre les deux indicateurs se creuse. Une carrière masculine «moyenne» mesurée à 39 ans représente alors 18,63 années d'expérience, mais seulement 17,28 années d'activité effective. La situation des hommes de 1962 se rapproche donc de celle des femmes de 1946. Mais dans le même temps, la situation des femmes a évolué : 18,36 années d'expérience ne correspondent alors plus qu'à 16,26 années d'activité effective. Plus que la réaction à ces variables des fonctions de gain, c'est bien leur valeur moyenne observée qui guide la modification des modèles de carrière individuelle. Une année d'expérience exerce donc toujours le même effet sur le salaire, à condition qu'il s'agisse d'expérience effective. Or, du fait de la multiplication des interruptions, l'écart se creuse entre expérience théorique (mesurée par la durée écoulée depuis la date d'entrée sur le marché du travail) et expérience effective. Traitant d'une question différente, mais effectuant le même changement de variable entre «expérience potentielle» et «expérience réelle», Meurs, Pailhe et Ponthieux (2008) aboutissent à un constat voisin. 
Graphique 1 : Chronogrammes du déroulement de carrière des hommes et des femmes des générations 1946 et 1962 de 14 à 39 ans

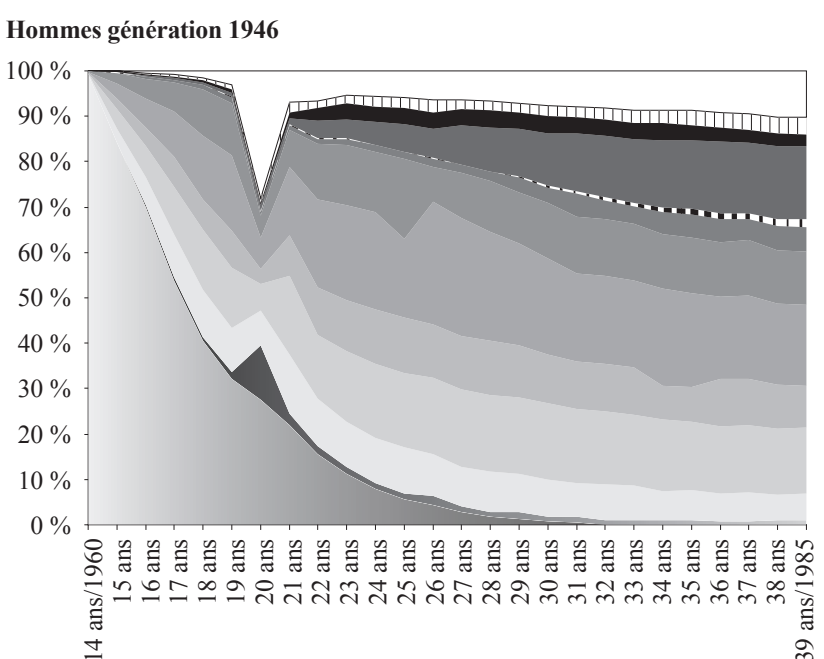

Femmes génération 1946

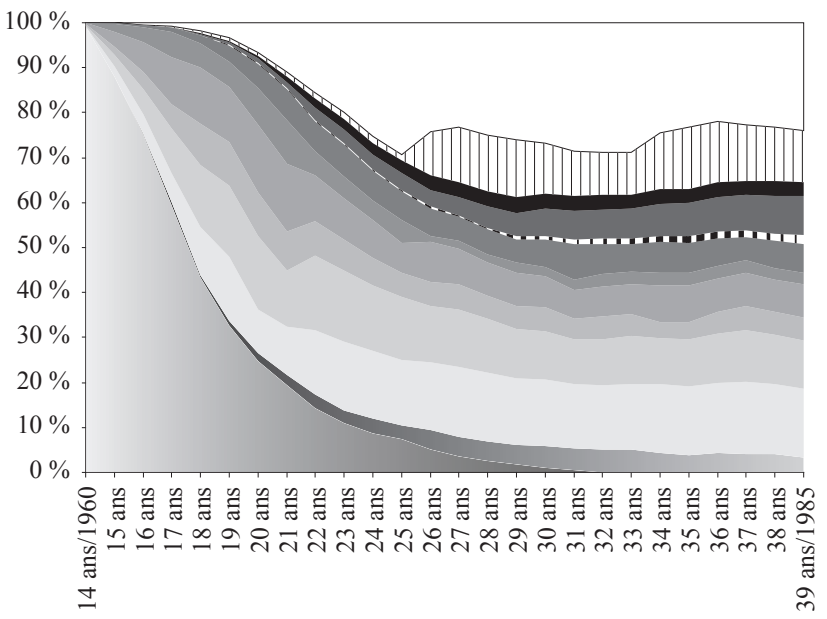

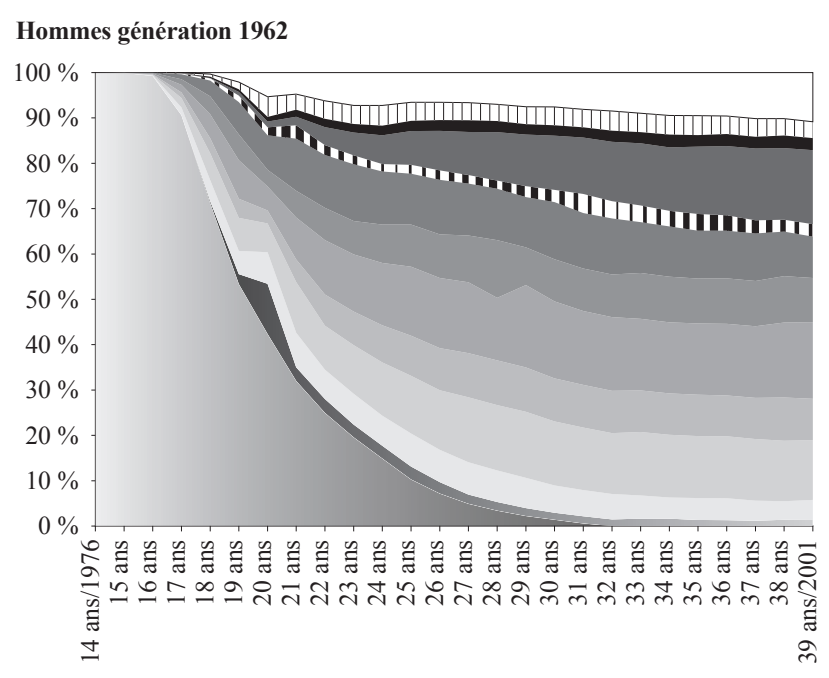

Femmes génération 1962

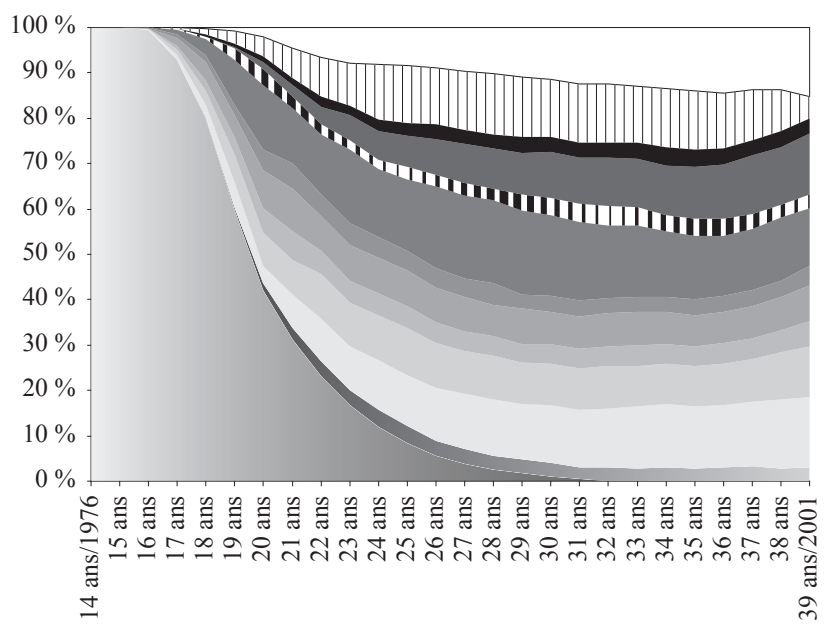

Source : DREES, EIC 2001 et calculs CNAM-INRA-AGIRC/ARRCO.

Tableau 3 : Générations 1946 et 1962, équations de salaire par sexe, modèle 1

\begin{tabular}{|c|c|c|c|c|c|c|c|c|}
\hline & \multicolumn{4}{|c|}{ Génération 1946} & \multicolumn{4}{|c|}{ Génération 1962} \\
\hline & \multicolumn{2}{|c|}{ Within } & \multicolumn{2}{|c|}{ Hausman-Taylor } & \multicolumn{2}{|c|}{ Within } & \multicolumn{2}{|c|}{ Hausman-Taylor } \\
\hline & Hommes & Femmes & Hommes & Femmes & Hommes & Femmes & Hommes & Femmes \\
\hline$E X P$ & $\begin{array}{c}0,164 \\
(0,001) \\
\end{array}$ & $\begin{array}{c}0,102 \\
(0,007)\end{array}$ & $\begin{array}{c}0,164 \\
(0,001)\end{array}$ & $\begin{array}{c}0,103 \\
(0,007)\end{array}$ & $\begin{array}{c}0,086 \\
(0,002) \\
\end{array}$ & $\begin{array}{c}0,031 \\
(0,008)\end{array}$ & $\begin{array}{c}0,087 \\
(0,002)\end{array}$ & $\begin{array}{c}0,032 \\
(0,008)\end{array}$ \\
\hline$E X P^{2}$ & $\begin{array}{l}-0,0033 \\
(0,00004)\end{array}$ & $\begin{array}{l}-0,0047 \\
(0,00025)\end{array}$ & $\begin{array}{l}-0,0033 \\
(0,00004)\end{array}$ & $\begin{array}{l}-0,0046 \\
(0,00024)\end{array}$ & $\begin{array}{l}-0,0020 \\
(0,00007)\end{array}$ & $\begin{array}{c}-0,00029 \\
(0,00030) n s\end{array}$ & $\begin{array}{l}-0,0021 \\
(0,00007)\end{array}$ & $\begin{array}{c}-0,00030 \\
(0,00030) n s\end{array}$ \\
\hline$C A D$ & $\begin{array}{c}0,553 \\
(0,006) \\
\end{array}$ & $\begin{array}{c}1,400 \\
(0,055) \\
\end{array}$ & $\begin{array}{c}0,553 \\
(0,006) \\
\end{array}$ & $\begin{array}{c}1,503 \\
(0,054) \\
\end{array}$ & $\begin{array}{c}0,498 \\
(0,008) \\
\end{array}$ & $\begin{array}{c}0,873 \\
(0,043) \\
\end{array}$ & $\begin{array}{c}0,548 \\
(0,008) \\
\end{array}$ & $\begin{array}{c}1,031 \\
(0,042) \\
\end{array}$ \\
\hline$E D U$ & & & $\begin{array}{c}0,066 \\
(0,001) \\
\end{array}$ & $\begin{array}{r}-0,051 \\
(0,011) \\
\end{array}$ & & & $\begin{array}{c}0,010 \\
(0,003)\end{array}$ & $\begin{array}{c}-0,024 \\
(0,014) n s\end{array}$ \\
\hline$A C C A N$ & $\begin{array}{l}-0,522 \\
(0,010) \\
\end{array}$ & $\begin{array}{c}-0,030 \\
(0,056) n s\end{array}$ & $\begin{array}{r}-0,522 \\
(0,010)\end{array}$ & $\begin{array}{c}-0,007 \\
(0,055) n s\end{array}$ & $\begin{array}{l}-0,225 \\
(0,008) \\
\end{array}$ & $\begin{array}{c}0,249 \\
(0,029) \\
\end{array}$ & $\begin{array}{r}-0,236 \\
(0,007)\end{array}$ & $\begin{array}{c}0,260 \\
(0,028)\end{array}$ \\
\hline ACCINDIV & & & $\begin{array}{l}-0,101 \\
(0,010)\end{array}$ & $\begin{array}{r}-0,978 \\
(0,090)\end{array}$ & & & $\begin{array}{r}-0,127 \\
(0,022)\end{array}$ & $\begin{array}{r}-0,649 \\
(0,124)\end{array}$ \\
\hline$R^{2}$ corrigé & 0,7246 & 0,0553 & & & 0,2104 & 0,0456 & & \\
\hline Individus & 8112 & 6864 & 8112 & 6864 & 10711 & 10217 & 10711 & 10217 \\
\hline Observations & 136500 & 98320 & 136500 & 98320 & 153780 & 139212 & 153780 & 139212 \\
\hline
\end{tabular}

Note : Les variables EXP, EXP² et EDU sont considérées comme endogènes. Les indicatrices annuelles sont inclues dans la régression mais les coefficients ne sont pas présentés. Les écarts-type estimés sont indiqués entre parenthèses. Tous coefficients significatifs au seuil de $1 \%$ sauf ns.

Source : DREES, EIC 2001 et calculs CNAM-INRA-AGIRC/ARRCO. 
Tableau 4 : Génération 1946 et 1962, équations de salaire par sexe, modèle 2

\begin{tabular}{|c|c|c|c|c|c|c|c|c|}
\hline & \multicolumn{4}{|c|}{ Génération 1946} & \multicolumn{4}{|c|}{ Génération 1962} \\
\hline & \multicolumn{2}{|c|}{ Within } & \multicolumn{2}{|c|}{ Hausman-Taylor } & \multicolumn{2}{|c|}{ Within } & \multicolumn{2}{|c|}{ Hausman-Taylor } \\
\hline & Hommes & Femmes & Hommes & Femmes & Hommes & Femmes & Hommes & Femmes \\
\hline$A N C$ & $\begin{array}{c}0,170 \\
(0,001)\end{array}$ & $\begin{array}{c}0,278 \\
(0,007)\end{array}$ & $\begin{array}{c}0,170 \\
(0,001)\end{array}$ & $\begin{array}{c}0,287 \\
(0,007)\end{array}$ & $\begin{array}{c}0,130 \\
(0,002)\end{array}$ & $\begin{array}{c}0,328 \\
(0,008)\end{array}$ & $\begin{array}{l}0,134 \\
(0002)\end{array}$ & $\begin{array}{c}0,339 \\
(0,008)\end{array}$ \\
\hline$A N C^{2}$ & $\begin{array}{c}-0,0038 \\
(0,00004)\end{array}$ & $\begin{array}{c}-0,0034 \\
(0,00024)\end{array}$ & $\begin{array}{c}-0,0038 \\
(0,00004)\end{array}$ & $\begin{array}{c}-0,0035 \\
(0,00025)\end{array}$ & $\begin{array}{c}-0,0029 \\
(0,00007)\end{array}$ & $\begin{array}{c}-0,0078 \\
(0,00032)\end{array}$ & $\begin{array}{l}-0,0030 \\
(0,00071)\end{array}$ & $\begin{array}{l}-0,0080 \\
(0,00031)\end{array}$ \\
\hline$C A D$ & $\begin{array}{c}0,547 \\
(0,006)\end{array}$ & $\begin{array}{c}1,171 \\
(0,054)\end{array}$ & $\begin{array}{c}0,568 \\
(0,005)\end{array}$ & $\begin{array}{c}1,258 \\
(0,052)\end{array}$ & $\begin{array}{c}0,482 \\
(0,008)\end{array}$ & $\begin{array}{c}0,736 \\
(0,043)\end{array}$ & $\begin{array}{c}0,522 \\
(0,008)\end{array}$ & $\begin{array}{c}0,873 \\
(0,041)\end{array}$ \\
\hline$E D U$ & & & $\begin{array}{c}0,068 \\
(0,001)\end{array}$ & $\begin{array}{c}0,150 \\
(0,010)\end{array}$ & & & $\begin{array}{c}0,045 \\
(0,002)\end{array}$ & $\begin{array}{c}0,166 \\
(0,013)\end{array}$ \\
\hline$A C C A N$ & $\begin{array}{c}-0,497 \\
(0,010)\end{array}$ & $\begin{array}{c}0,414 \\
(0,055)\end{array}$ & $\begin{array}{r}-0,508 \\
(0,010)\end{array}$ & $\begin{array}{c}0,448 \\
(0,053)\end{array}$ & $\begin{array}{l}-0,189 \\
(0,007)\end{array}$ & $\begin{array}{c}0,300 \\
(0,028)\end{array}$ & $\begin{array}{l}-0,197 \\
(0,007)\end{array}$ & $\begin{array}{c}0,314 \\
(0,028)\end{array}$ \\
\hline ACCINDIV & & & $\begin{array}{r}-0,062 \\
(0,010)\end{array}$ & $\begin{array}{r}-0,710 \\
(0,085)\end{array}$ & & & $\begin{array}{l}-0,070 \\
(0,021)\end{array}$ & $\begin{array}{c}-0,512 \\
(0,121)\end{array}$ \\
\hline $\mathbf{R}^{2}$ corrigé & 0,7232 & 0,0877 & & & 0,2263 & 0,0583 & & \\
\hline Individus & 8112 & 6864 & 8112 & 6864 & 10711 & 10217 & 10711 & 10217 \\
\hline Observations & 136500 & 98320 & 136500 & 98320 & 153780 & 139212 & 153780 & 139212 \\
\hline
\end{tabular}

Note : Les variables ANC, $\mathrm{ANC}^{2}$ et EDU sont considérées comme endogènes. Les indicatrices annuelles sont inclues dans la régression mais les coefficients ne sont pas présentés. Les écarts-type estimés sont indiqués entre parenthèses. Tous coefficients significatifs au seuil de $1 \%$.

Source : DREES, EIC 2001 et calculs CNAM-INRA-AGIRC/ARRCO.

\section{Différents types de carrière salariale}

L'analyse typologique fait apparaître douze classes pour la génération 1946. Ces classes se retrouvent pour la génération 1962, à deux exceptions près : une classe de la génération 1946 disparaît tandis qu'une nouvelle classe apparaît pour la génération 1962. En comparant ces douze classes entre les générations 1946 et 1962, on constate la progression du nombre de salariés concernés par des carrières longues dans le secteur privé : leur part passe de 53,2\% dans la génération née en 1946 au tiers $(66,7 \%)$ de la génération née en 1962 (4). Les femmes deviennent de plus en plus concernées par ces carrières longues : $56,5 \%$ des femmes de la génération 1946 et $62,5 \%$ de la génération 1962, confortant le résultat de Dominique Meurs et Sophie Ponthieux (2006) selon lequel les femmes entre 30 et 50 ans ont des carrières de plus en plus complètes au fil des générations.

Parmi ces douze classes, nous en retenons sept pour une analyse plus approfondie. La sélection de ces sept classes résulte d'un triple critère : leur poids dans la génération considérée, la part de femmes correspondante dans la génération, le poids des femmes dans la classe. Leurs caractéristiques sont présentées dans le tableau 5 et les équations de gain correspondantes dans le tableau 6. L'analyse de ces classes révèle quatre évolutions majeures entre les générations 1946 et 1962 : aux deux extrémités de l'échelle des salaires, la forte progression des carrières à bas ou très bas mais aussi celle des carrières à hauts ou très hauts salaires, la progression/persistance des carrières marquées par différentes formes de précarité, et le déclin du modèle "classique» de la carrière féminine des années 1950 et 1960. 
Tableau 5 : Caractéristiques descriptives des sept classes retenues

\begin{tabular}{|c|c|c|c|c|c|c|c|c|}
\hline $\begin{array}{c}\text { Classe de la } \\
\text { typologie }\end{array}$ & Génération & $\begin{array}{c}\text { Salaire } \\
\text { annuel } \\
\text { moyen } \\
\text { (écart-type) }\end{array}$ & $\begin{array}{c}\text { Accident } \\
\text { dans l'année } \\
\text { (\% de } \\
\text { salariés) }\end{array}$ & $\begin{array}{l}\text { Accident } \\
\text { dans la } \\
\text { carrière } \\
(\% \text { de } \\
\text { salariés })\end{array}$ & $\begin{array}{l}\text { Expérience, } \\
\text { (ancienneté) } \\
\text { (en années) }\end{array}$ & $\begin{array}{c}\text { Cadres } \\
(\%)\end{array}$ & $\begin{array}{c}\text { Femmes } \\
(\%)\end{array}$ & \begin{tabular}{|c|} 
Poids de la \\
classe dans la \\
génération \\
$(\%$ de \\
salariés $)$ \\
\end{tabular} \\
\hline \multirow{3}{*}{$\begin{array}{l}\mathrm{C} 2 \text { : Carrières } \\
\text { longues à très bas } \\
\text { salaires }\end{array}$} & 1946 & 1 & 1 & I & 1 & 1 & 1 & I \\
\hline & $\mathrm{N}=0$ & & & & & & & \\
\hline & $\begin{array}{c}1962 \\
N=955\end{array}$ & $\begin{array}{l}10703 \\
(8004)\end{array}$ & 5,3 & 91,6 & $\begin{array}{c}19,6 \\
(18,2)\end{array}$ & 0,7 & 79,7 & 4,5 \\
\hline \multirow{2}{*}{$\begin{array}{l}\mathrm{C} 3 \text { : Carrières } \\
\text { longues à bas } \\
\text { salaires }\end{array}$} & $\begin{array}{c}1946 \\
N=1997\end{array}$ & $\begin{array}{l}12717 \\
(5762)\end{array}$ & 1,0 & 83,5 & $\begin{array}{c}22,1 \\
(21,8)\end{array}$ & 0,4 & 33,2 & 13,2 \\
\hline & $\begin{array}{c}1962 \\
N=3108\end{array}$ & $\begin{array}{l}15153 \\
(5645) \\
\end{array}$ & 4,1 & 88,2 & $\begin{array}{c}20,3 \\
(19,5)\end{array}$ & 1,0 & 41,5 & 14,6 \\
\hline \multirow{2}{*}{$\begin{array}{l}\mathrm{C} 5: \text { Carrières } \\
\text { longues à hauts } \\
\text { salaires }\end{array}$} & $\begin{array}{c}1946 \\
N=1426\end{array}$ & $\begin{array}{l}18209 \\
(7171)\end{array}$ & 0,7 & 74,5 & $\begin{array}{c}20,9 \\
(20,9)\end{array}$ & 1,9 & 23,4 & 9,4 \\
\hline & $\begin{array}{c}1962 \\
N=2961\end{array}$ & $\begin{array}{l}22787 \\
(9148)\end{array}$ & 2,7 & 84,8 & $\begin{array}{c}19,8 \\
(19,3)\end{array}$ & 9,0 & 31,2 & 13,9 \\
\hline \multirow{2}{*}{$\begin{array}{l}\text { C6 : Carrières } \\
\text { longues à très } \\
\text { hauts salaires }\end{array}$} & $\begin{array}{c}1946 \\
N=1369\end{array}$ & $\begin{array}{c}26774 \\
(16836)\end{array}$ & 0,9 & 77,7 & $\begin{array}{c}20,4 \\
(20,0)\end{array}$ & 52,6 & 15,8 & 9,1 \\
\hline & $\begin{array}{c}1962 \\
N=1348\end{array}$ & $\begin{array}{c}37089 \\
(21529)\end{array}$ & 2,9 & 88,2 & $\begin{array}{c}18,5 \\
(17,4)\end{array}$ & 66,1 & 27,7 & 6,3 \\
\hline \multirow{2}{*}{$\begin{array}{l}\mathrm{C} 1 \text { : Carrières } \\
\text { longues avec des } \\
\text { interruptions et/ } \\
\text { ou des très bas } \\
\text { salaires }\end{array}$} & $\begin{array}{c}1946 \\
N=3259\end{array}$ & $\begin{array}{c}9884 \\
(6975) \\
\end{array}$ & 2,5 & 93,9 & $\begin{array}{c}20,7 \\
(17,7)\end{array}$ & 1,6 & 62,6 & 21,6 \\
\hline & $\begin{array}{c}1962 \\
N=4718\end{array}$ & $\begin{array}{l}12852 \\
(8864)\end{array}$ & 12,2 & 98,3 & $\begin{array}{c}18,3 \\
(14,5)\end{array}$ & 3,3 & 59,8 & 22,2 \\
\hline \multirow[t]{2}{*}{$\begin{array}{l}\mathrm{C} 11 \text { : Carrières } \\
\text { très précaires }\end{array}$} & $\begin{array}{c}1946 \\
\mathrm{~N}=702\end{array}$ & $\begin{array}{c}4752 \\
(8318) \\
\end{array}$ & 1,2 & 93,1 & $\begin{array}{c}20,3 \\
(16,5) \\
\end{array}$ & 0,5 & 88,1 & 4,7 \\
\hline & $\begin{array}{c}1962 \\
N=976\end{array}$ & $\begin{array}{c}5900 \\
(10219) \\
\end{array}$ & 0,4 & 92,5 & $\begin{array}{c}19,1 \\
(17,2)\end{array}$ & 1,1 & 92,5 & 4,6 \\
\hline \multirow{2}{*}{$\begin{array}{l}\text { C13: Carrières } \\
\text { courtes débou- } \\
\text { chant sur } \\
\text { l'inactivité }\end{array}$} & $\begin{array}{c}1946 \\
N=1874\end{array}$ & $\begin{array}{c}7511 \\
(46169) \\
\end{array}$ & 2,0 & 90,2 & $\begin{array}{c}14,1 \\
(11,0)\end{array}$ & 8,7 & 75,1 & 12,4 \\
\hline & $\begin{array}{c}1962 \\
N=1518\end{array}$ & $\begin{array}{l}10905 \\
(9029)\end{array}$ & 9,6 & 94,2 & $\begin{array}{l}11,2 \\
(8,0)\end{array}$ & 2,7 & 59,7 & 7,2 \\
\hline \multirow[t]{2}{*}{$\begin{array}{l}\text { Ensemble de la } \\
\text { population }\end{array}$} & $\begin{array}{c}1946 \\
N=15099\end{array}$ & $\begin{array}{c}14365 \\
(16308) \\
\end{array}$ & 1,5 & 83,4 & $\begin{array}{c}19,8 \\
(18,5)\end{array}$ & 7,5 & 49,3 & 100 \\
\hline & $\begin{array}{c}1962 \\
N=21313\end{array}$ & $\begin{array}{c}17638 \\
(13019) \\
\end{array}$ & 6,1 & 90,4 & $\begin{array}{c}18,5 \\
(16,9)\end{array}$ & 9,1 & 49,9 & 100 \\
\hline
\end{tabular}

Source : DREES, EIC 2001 et calculs CNAM-INRA-AGIRC/ARRCO.

Tableau 6 : Générations 1946 et 1962, équations de salaire par classe de la typologie

\begin{tabular}{|l|c|c|c|c|c|c|}
\hline \multirow{2}{*}{ Classe } & \multicolumn{3}{|c|}{ Génération 1946 } & \multicolumn{3}{c|}{ Génération 1962 } \\
\cline { 2 - 6 } & Constante & EXP & EXP & Constante & EXP & EXP \\
\hline $\begin{array}{l}\text { C2 }: \text { Carrières longues à très bas } \\
\text { salaires }\end{array}$ & & & & 9,06 & 0,104 & $-0,004$ \\
\hline C3: Carrières longues à bas salaires & 7,48 & 0,165 & $-0,003$ & 8,93 & 0,103 & $-0,003$ \\
\hline $\begin{array}{l}\text { C5: Carrières longues à hauts } \\
\text { salaires }\end{array}$ & 8,03 & 0,177 & $-0,007$ & 9,27 & 0,104 & $-0,003$ \\
\hline $\begin{array}{l}\text { C6: Carrières longues à très hauts } \\
\text { salaires }\end{array}$ & 9,02 & 0,158 & $-0,004$ & 10,52 & 0,056 & $-0,003$ \\
\hline $\begin{array}{l}\text { C1: Carrières longues avec des inter- } \\
\text { ruptions et/ou des très bas salaires }\end{array}$ & 7,58 & 0,105 & $-0,002$ & 8,77 & 0,025 & 0,001 \\
\hline C11: Carrières très précaires & 6,05 & $-0,076$ & $-0,006$ & 8,54 & 0,080 & $\mathrm{~ns}$ \\
\hline $\begin{array}{l}\text { C13: Carrières courtes débouchant } \\
\text { sur 1'inactivité }\end{array}$ & 7,78 & 0,156 & $-0,008$ & 9,06 & 0,091 & $-0,002$ \\
\hline
\end{tabular}

Note : Estimations en Within, plus indicatrices annuelles. Tous coefficients significatifs au seuil de $1 \%$, sauf ns.

Source : DREES, EIC 2001 et calculs CNAM-INRA-AGIRC/ARRCO. 


\section{La forte progression des carrières longues à bas ou très bas salaires}

Les carrières longues à bas salaires (inférieurs aux 45 premiers centiles de salaire) étaient déjà présentes dans la génération 1946 ( $c f$. graphique 3 ). Mais leur part augmente dans la génération 1962, tant du fait de la croissance de la part de la classe C3 dans la population totale de la génération (de 13,2 à 14,6\%) que de l'apparition d'une nouvelle classe (C2) aux caractéristiques encore plus affirmées (salaires inférieurs aux 20 premiers centiles, $c f$. graphique 2). Les fonctions de gain associée à ces deux classes présentent des formes classiques de ce type de carrière : faible niveau de départ mais également marge de progression réduite, en particulier pour la génération la plus récente.
Dans la nouvelle classe, le salaire annuel brut moyen est de 10703 euros, ce qui est effectivement très bas pour la génération 1962. La part de salariés de la classe concernés par des interruptions est élevée ce qui situe cette classe à la limite des carrières précaires ( $c f$. infra). Pour la classe $\mathrm{C} 3$, déjà présente dans la génération 1946, les niveaux de salaire annuel brut moyens s'établissent à 12717 euros (génération 1946) et à 15553 (génération 1962). La proportion de cadres est quasiment nulle ( $c f$. tableau 5).

La composition entre hommes et femmes tend à s'équilibrer dans la classe ancienne : il s'agissait d'une classe formée aux deux tiers d'hommes dans la génération 1946, ils ne sont plus que $54 \%$ dans la génération 1962. La part des femmes se situe d'emblée à un niveau très élevé dans la nouvelle classe, près de $80 \%$ ( $c f$. tableau 5$)$, si bien que l'on peut parler d'une classe féminine.

\section{Graphique 2 : carrières longues à très bas salaire}

1946 : la classe n'apparaît pas

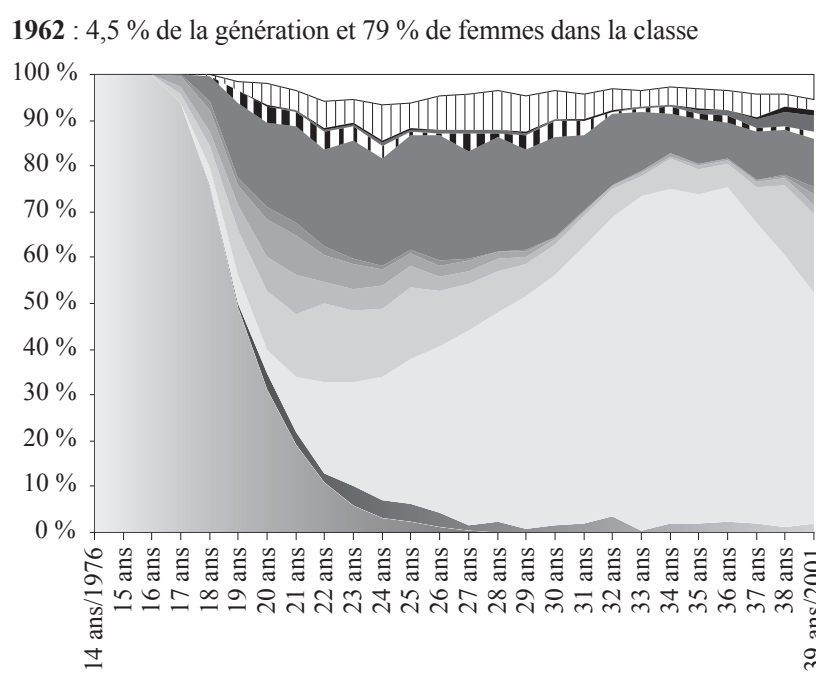

Source : DREES, EIC 2001 et calculs CNAM-INRA-AGIRC/ARRCO.

Graphique 3 : carrières longues à bas salaires

$1946: 13,2 \%$ de la génération et $34 \%$ de femmes dans la classe

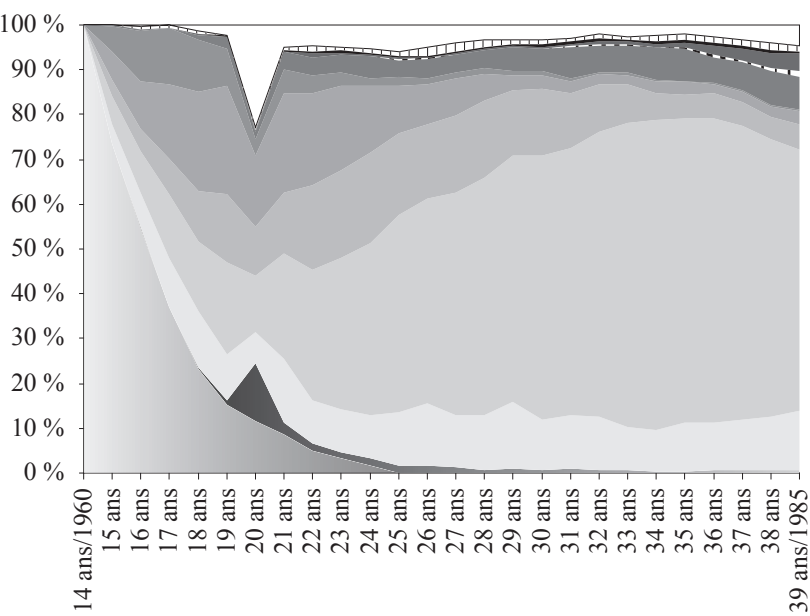

1962 : $14,6 \%$ de la génération et $42 \%$ de femmes dans la classe

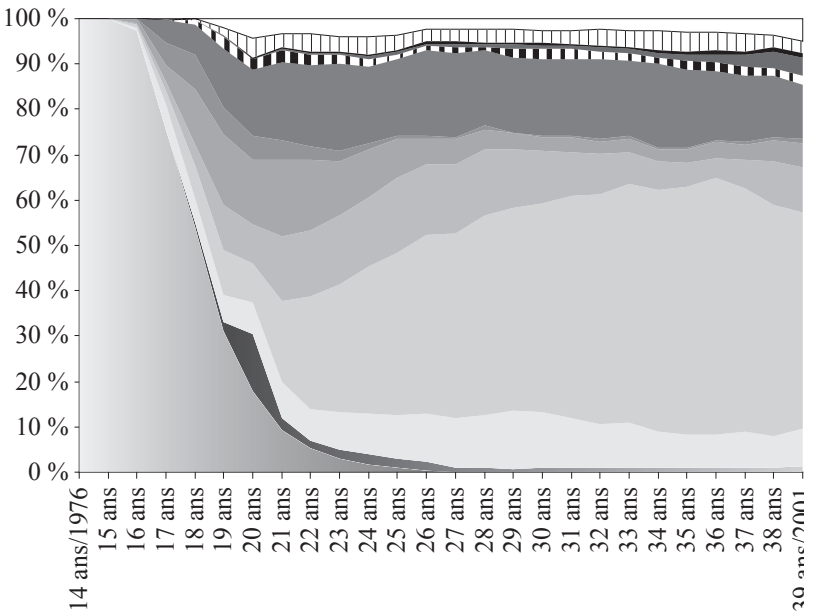

Source : DREES, EIC 2001 et calculs CNAM-INRA-AGIRC/ARRCO. 


\section{La progression des carrières longues à hauts et très hauts salaires}

À l'autre extrémité de l'échelle des salaires se trouvent les carrières longues à hauts et très hauts salaires (entre les $60^{\mathrm{e}}$ et $85^{\mathrm{e}}$ centiles de salaire et au-delà du $85^{\mathrm{e}}$ centile) dont le poids progresse entre les générations 1946 et 1962 pour atteindre le $5^{\mathrm{e}}$ de la génération née en 1962. Les fonctions de gain suggèrent un fort potentiel de croissance du salaire pour la génération née en 1946, qui s'atténue assez largement pour celle née en 1962. Les niveaux moyens de salaire annuel brut demeurent nettement différents entre les deux classes : 18209 euros (génération 1946) et 22787 euros (génération 1962) pour les hauts, 26774 euros (génération 1946) et 37089 euros (génération 1962) pour les très hauts salaires. La progression salariale de ces derniers est d'ailleurs plus importante. Le statut de cadre différencie également ces deux classes. Il est fréquent $(52,6 \%$ et $66,1 \%)$ chez les très hauts salaires, et assez rare $(1,9 \%$ et $9 \%)$ chez les hauts. En revanche ces catégories partagent la même stabilité : il y a peu d'interruptions au regard de la moyenne de la population mais leur nombre augmente quand même d'une génération à l'autre ( $c f$. graphiques 4 et 5 ). Les individus à très hauts salaires possèdent une expérience et une ancienneté plus faibles du fait d'une entrée plus tardive sur le marché du travail (respectivement à l'âge de 22 ans et 24 ans). Ils ont donc a priori un niveau d'éducation initiale plus élevé. Il s'agit de deux classes semblables sur bien des points comme la stabilité dans l'emploi, même si celle-ci s'atténue, mais avec des niveaux quantitatifs (salaire) et qualitatif (accès au statut cadre) très différents.

Le poids des femmes dans ces deux catégories était faible dans le cas de la génération 1946. Il augmente dans le cas de la génération 1962, mais reste très inférieur à la moyenne de la population. Une certaine convergence apparaît donc aussi par le haut, mais elle est d'autant plus lente que le niveau de salaire s'élève : la part des femmes est de 31,2\% dans les hauts salaires et $27,7 \%$ dans les très hauts, confortant l'idée du plafond de verre.

De façon plus générale la progression de ces carrières à hauts salaires et la présence croissante des femmes en leur sein confirme les résultats récents de Casey Muldigan et Yona Rubinstein (2008). En comparant la situation américaine des années 1975-1979 à celle des années 1995-1999, les auteurs montrent que la réduction apparente de l'écart salarial entre hommes et femmes s'explique aussi par la croissance des inégalités salariales entre femmes et l'existence de hauts salaires féminins.

\section{Graphique 4 : carrières longues à hauts salaires}

1946 : 9,4 \% de la génération et $23,4 \%$ de femmes dans la classe

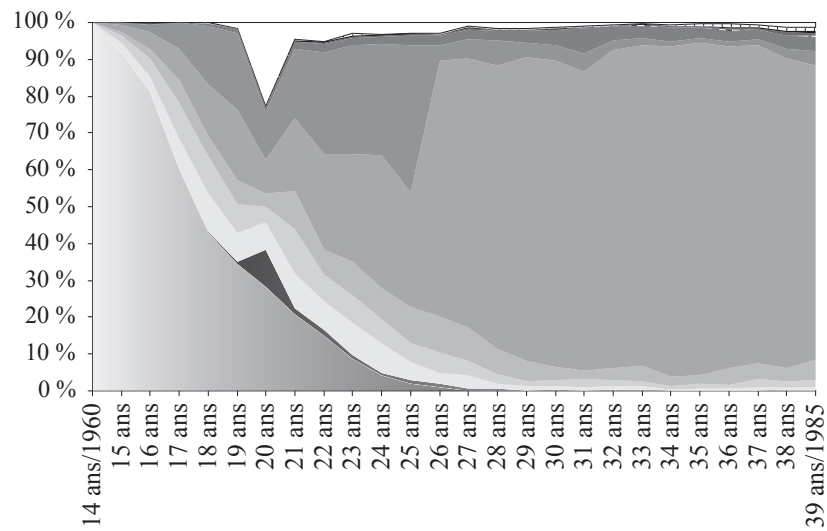

$1962: 13,9 \%$ de la génération et $31,5 \%$ de femmes dans la classe

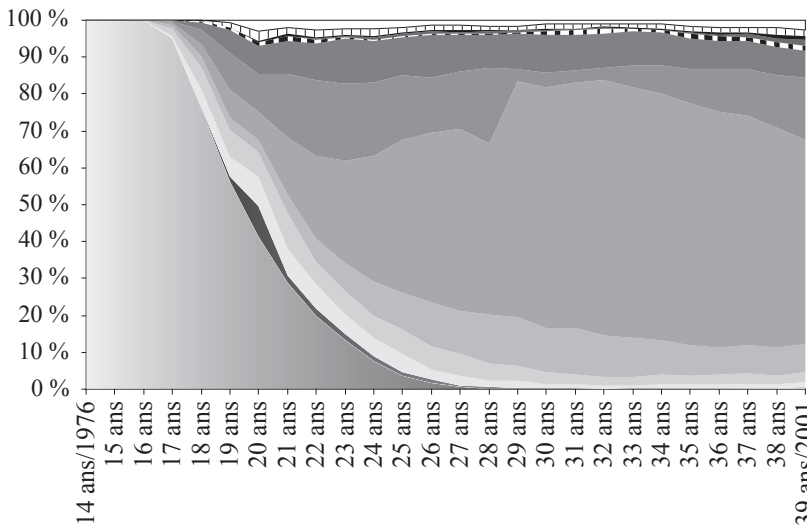

Source : DREES, EIC 2001 et calculs CNAM-INRA-AGIRC/ARRCO.

\section{Graphique 5 : carrières longues à très hauts salaires}
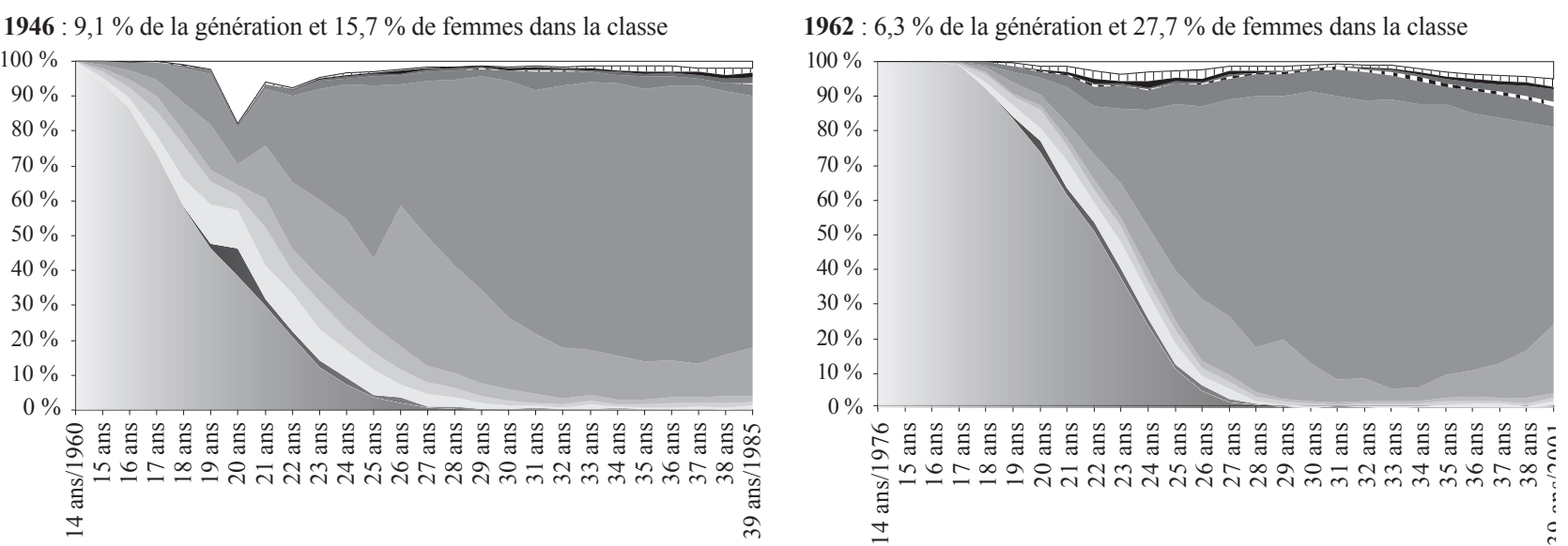

Source : DREES, EIC 2001 et calculs CNAM-INRA-AGIRC/ARRCO. 


\section{La progression/persistance des carrières marquées par différentes formes de précarité}

Deux classes de la typologie, présentes aussi bien dans la génération 1946 que dans celle de 1962, illustrent la progression des carrières marquées par différentes formes de précarité.

Une première classe $(\mathrm{C} 1)$ est constituée de salariés poursuivant des carrières longues et continues dans le secteur privé mais marquées à la fois par des interruptions et/ou des très bas salaires ( $c f$. graphique 6). Son poids était déjà élevé dans la génération 1946 et il augmente encore dans la génération 1962. C'est près du quart des salariés observés qui sont concernés. Cette classe est marquée dès 1946 par la conjonction de très bas niveaux de salaire et de très fréquentes interruptions de l'activité sans que le lien à l'emploi ne soit rompu, ni la validation de quatre trimestres annuels pour la retraite. Le salaire annuel brut moyen est faible, 9884 et 12852 euros respectivement pour les générations 1946 et 1962, soit environ $50 \%$ en dessous des valeurs moyennes de l'ensemble de la population. La fonction de gain présente un profil classique mais qui tend à s'aplatir dans le cas de la génération 1962. La fréquence des interruptions est remarquablement élevée et croît pour s'établir à un niveau de plus de $12 \%$ des observations. Il en découle logiquement un creusement de l'écart entre expérience théorique et ancienneté effective. Le nombre de femmes dans cette classe a progressé mais leur part relative diminue, tout en restant majoritaire. La conjonction entre précarité et très bas salaire, telle qu'elle est présentée par cette classe, continue à concerner majoritairement les femmes mais également de plus en plus d'hommes. Dans le même temps cette précarité s'accroît dans la population, tant du point de vue du nombre de salariés concernés, que de la fréquence des interruptions d'activité.
Une seconde classe $(\mathrm{C} 11)$ révèle une forme différente de précarité massivement féminine (cf. graphique 7). Les salaires perçus y sont très faibles : 4752 euros pour la génération 1946 et 5900 euros pour la génération 1962, soit le tiers de la moyenne de la population d'ensemble. Les interruptions d'activité, en revanche, n'y sont pas exceptionnellement fréquentes et elles n'augmentent pas. Il s'agit d'une population qui poursuit une carrière à la limite de l'activité professionnelle avec des temps partiels ou la succession de CDD de courte durée par exemple. Sa précarité s'illustre par le fait qu'elle ne valide pas quatre trimestres par an pour sa retraite. L'observation des fonctions de gain montre que le modèle canonique y est complètement inadapté. Presque exclusivement féminine (autour de neuf salariées sur dix), cette classe témoigne d'une forme de précarité propre aux femmes dont l'apparition n'est pas récente.

\section{Le déclin du modèle "classique" de la carrière féminine des années 1950 et 1960}

Un des modèles de carrière professionnelle féminine des années 1950 et 1960, celui où les femmes s'arrêtent quasi définitivement de travailler à la naissance de leurs enfants, décline sous cette forme et se transforme ( $c f$. graphique 8 ). Ce modèle est fort dans la génération 1946 où l'on constate un retrait du marché du travail à partir de l'âge de 25-26 ans pour observer ensuite une légère reprise de l'activité professionnelle à partir de 35 ans. Il concerne principalement des femmes qui ont quitté le marché du travail très jeune, ce qui laisse supposer une sortie d'activité en raison de la maternité et de l'éducation des enfants. Pour une analyse détaillée de l'impact sur la carrière salariale des femmes de ces interruptions associées à la présence des enfants, on consultera Meurs, Pailhe et Ponthieux (2008). 


\section{Graphique 6 : carrières longues avec des interruptions et/ou des très bas salaires}

$1946: 21,6 \%$ de la génération et $65,6 \%$ de femmes dans la classe

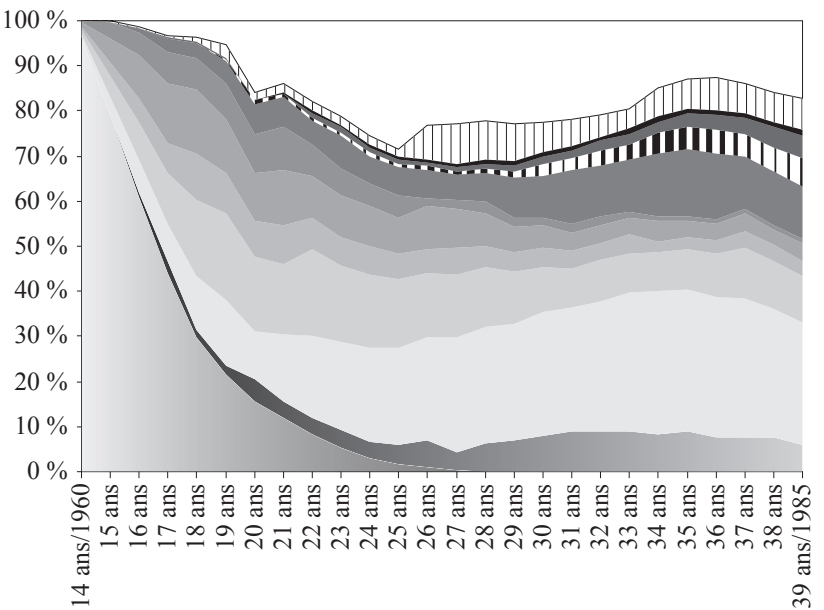

1962 : $22,2 \%$ de la génération et $59 \%$ de femmes dans la classe

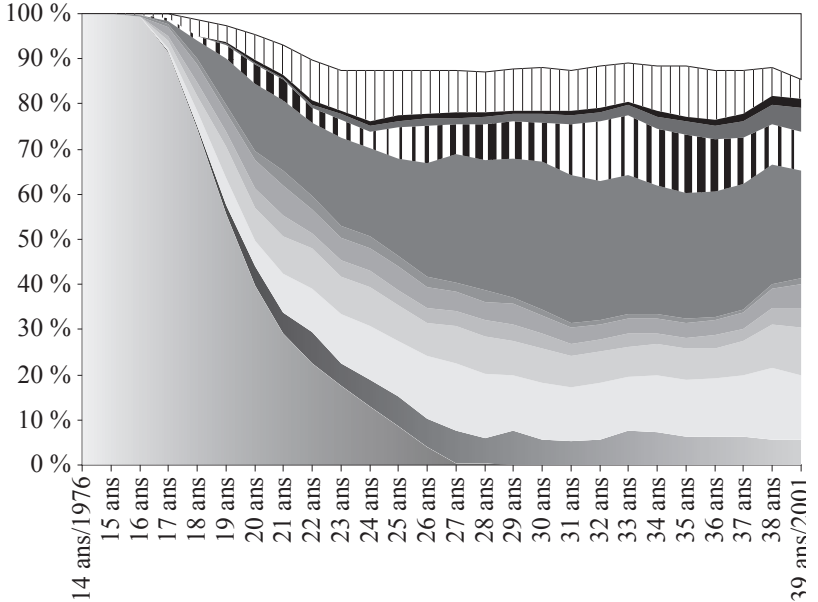

Source : DREES, EIC2001 et calculs CNAM-INRA-AGIRC/ARRCO.

Graphique 7 : carrières très précaires avec moins de quatre trimestres validés

$1946: 4,7 \%$ de la génération et $81,7 \%$ de femmes dans la classe

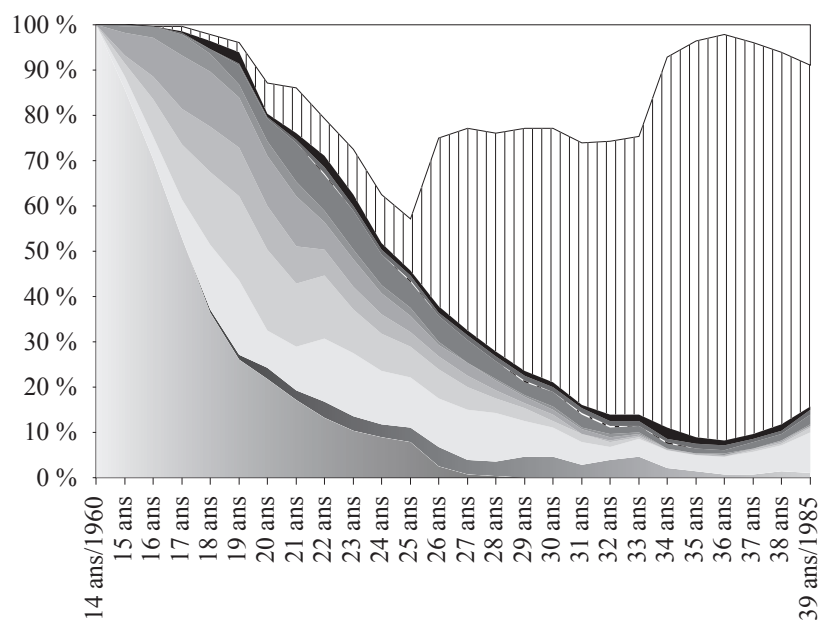

$1962: 4,6 \%$ de la génération et $85,9 \%$ de femmes dans la classe

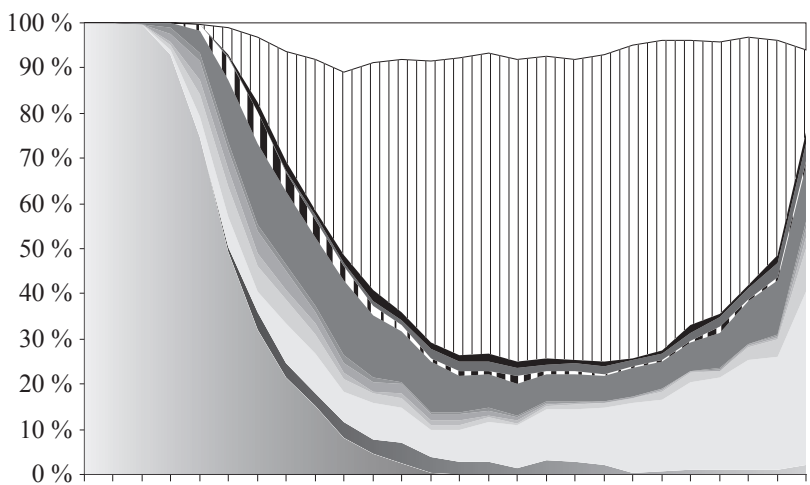

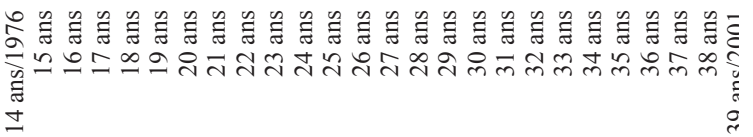

Source : DREES, EI C2001 et calculs CNAM-INRA-AGIRC/ARRCO.
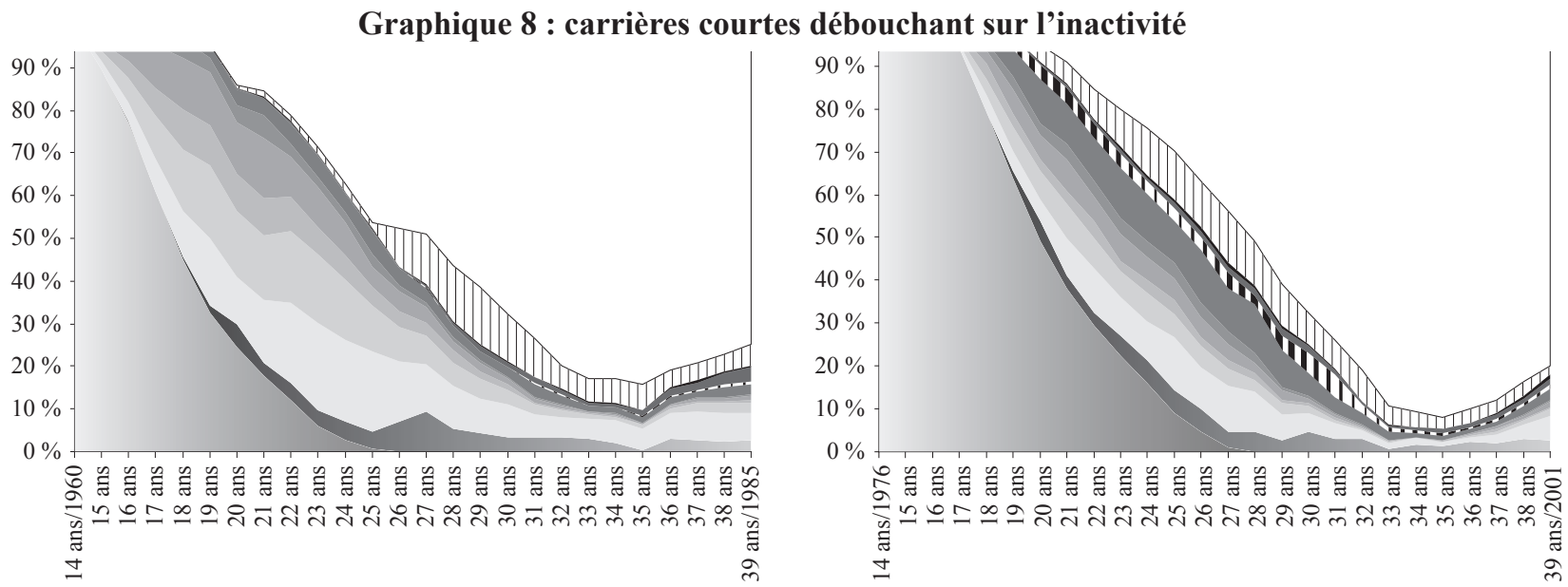

Source : DREES, EI C2001 et calculs CNAM-INRA-AGIRC/ARRCO. 
Ce modèle existe toujours dans la génération 1962 mais il concerne une part moins importante des salariés $(7,2 \%$ contre $12,4 \%)$. De plus, son profil évolue assez sensiblement. Les fonctions de gain suggèrent une carrière beaucoup plus plate pour la génération 1962. Le taux de cadres, 8,7 \%, dans la génération 1946 chute à $2,7 \%$. La durée moyenne d'expérience, déjà faible $(14,12$ ans) dans la génération 1946 , tombe à 10,98 ans pour la génération 1962. La durée effective d'activité connaît la même évolution, elle descend de 11,16 à 7,98 ans. Les taux d'interruption d'activité sont bien sûr élevés, même s'il s'agit d'interruptions particulières, longues et non répétitives, essentiellement un passage quasi irréversible à l'inactivité. En même temps que ce modèle évolue, sa spécificité féminine s'atténue : la part des femmes baisse de $75,1 \%$ à $59,7 \%$. Les hommes deviennent de plus en plus concernés par ce type de carrière, vraisemblablement pour des raisons qui ne sont pas toutes liées à l'éducation des enfants. Il s'agit là d'une dernière illustration d'une convergence de la forme des carrières entre les hommes et les femmes, selon un modèle différent de celui de la génération précédente.

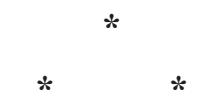

Les carrières des salariés du secteur privé ont connu une profonde évolution entre la génération des individus nés en 1946 et celle des individus nés en 1962. La différenciation entre la carrière des hommes et celle des femmes constitue une clef de lecture efficace pour rendre compte de la situation initiale, celle du marché du travail des années 1960 à 1975. Cette clé de lecture est beaucoup moins efficace pour les années plus récentes. L'utilisation d'un outil classique comme l'estimation de fonctions de gain suggère que le modèle canonique d'une carrière ascendante sans trop de discontinuités n'a existé que pour les hommes de la génération 1946. Il ne caractérise qu'imparfaitement la situation des femmes de cette génération et encore moins celle de chacun, homme ou femme, dans la génération 1962. La croissance du nombre des interruptions d'activité est telle qu'il convient plus que jamais de raisonner en termes d'ancienneté dans l'activité effective plutôt qu'en termes d'expérience théorique liée à la date d'entrée sur le marché du travail.

La construction d'une typologie des carrières individuelles permet d'enrichir considérablement ce diagnostic. Une partie importante (près du tiers) du salariat est concernée par des carrières précaires. La précarité peut prendre plusieurs formes : des interruptions fréquentes de l'activité éventuellement associées à des niveaux de salaire faible ou une situation de sous-emploi, marqué par un lien permanent mais extrêmement distendu à l'activité. Ces situations illustrent de façon complémentaire la différenciation entre les carrières des hommes et celles des femmes. La première concerne toujours majoritairement des femmes mais aussi de plus en plus d'hommes. La seconde, qui existait déjà pour la génération née en 1946 est l'apanage quasi exclusif des femmes. Les carrières à bas (respectivement très bas) et haut (respectivement très haut) salaires continuent de se différencier nettement. La relation est quasi monotone entre le niveau de salaire et la part des femmes dans la classe considérée. Considérons cependant que toutes ces catégories sont marquées par un même phénomène : l'accroissement du nombre moyen d'interruptions de carrière. Enfin, certaines formes de carrières féminines, comme les carrières écourtées pour cause de maternité et d'éducation des enfants, voient à la fois leur poids relatif et leur spécificité féminine se réduire.

En conclusion, une forme de convergence semble bien s'opérer entre carrières masculines et féminines mais sur un modèle nouveau, sans doute plus proche de la situation initiale des secondes que du modèle canonique de la carrière masculine. Précarité et discontinuité accrues, maintien d'un éventail salarial large qui relie négativement le niveau de salaire d'une catégorie à la part des femmes qui la compose : tels semblent en être les maîtres mots. 


\section{Bibliographie}

Amossé T., Ben Halima M.A. (2010), «Mobilité et stabilité sur le marché du travail : une dualisation en trompe-l'œil» Connaissance de l'emploi, le Quatre Pages du CEE no 75, décembre.

Behaghel L. (2003), «Insécurité de l'emploi : le rôle protecteur de l'ancienneté a-t-il baissé en France?», Économie et Statistique no 348, pp. 61-79.

Behaghel L. (2006), Lire l'économétrie, édition La Découverte.

Berton F, Huiban J.P., Nortier F. (2009), «Génération 1946 versus génération 1962 : vers des carrières salariales plus discontinues» in BARNAY T. \& LEGENDRE F. (dir.) «Emploi et politiques sociales, trajectoires d'emploi et rémunérations », L’Harmattan, tome 2, pp. 7-21.

BRIARD K. (2007), «Profils type des salariés du secteur privé : approche par une classification des carrières», Économie et Prévision no 180-181, 2007/4-5, pp. 59-85.

Buchinsky M., Fougère D., Kramarz F. (1998), «La mobilité salariale en France : 1967-1987», Revue Économique vol 49 no 3, pp. 879-890.

CARD D. (1999), "The causal effect of education on earnings", in Ashenfelter O. and Layard R. eds. Handbook of Labor Economics, vol. 3, North Holland.

Colin C., El Mekkaoui de Freitas N., Michaudon H. (2004), «Comment mesurer les droits acquis à la retraite en France : la création de l'Échantillon Interrégimes de Cotisants », Courrier des Statistiques n ${ }^{\circ} 111$, septembre, pp. 19-29.

Cornwell C., Rupert P. (1988), “Efficient Estimation with Panel data: an empirical comparison of instrumental Variables estimators", Journal of Applied Econometrics no 3, pp. 149-155.

Debrand T., Privat A.G. (2002), «L'évolution des carrières salariales au cours des cinquante dernières années », Retraite et Société no 36, pp. 188-201.

Demailly D., Le Minez S. (1999), «Les salariés à temps complet au voisinage du Smic de 1976 à 1996 », INSEE, Insee Première, $\mathrm{n}^{\circ} 642$.

Givord P., Maurin E. (2003), «La montée de l'instabilité professionnelle et ses causes», Revue économique vol. 54 no 3, pp. 617-626.
Grelet Y. (2002), «Des typologies de parcours méthodes et usages », Céreq, Notes de travail génération $92 \mathrm{n}^{\circ} 20$, juillet, $47 \mathrm{p}$.

Hausman J.A., TAYlor W. (1981), "Panel data and unobservable individual effects", Econometrica, no 46, pp. 1377-1399.

De Larquier G., Remillon D. (2008), «Assiste-t-on à une transformation uniforme des carrières professionnelles vers plus de mobilité?», Travail et Emploi no 113, janvier-mars, pp. 13-30

LeClair M., Roux S. (2006), «Les emplois de courte durée dans les entreprises », Données Sociales. La société française, pp. 25-253.

LeClair M., Roux S. (2007), "Productivité relative et utilisation des emplois de courte durée dans les entreprises», Économie et Statistique, no 405/406, pp. 47-76.

Lollivier S., Payen J.-F. (1990), «L’hétérogénéité des carrières individuelles mesurée sur données de panel», Économie et Prévision no 92-93, pp. 87-95.

Magnac T., Roux S. (2009), «Dynamique des salariés dans une cohorte », Économie et Prévision, n 1, pp. 1-24.

Meurs D., Ponthieux S. (2006), «L'écart des salaires entre les hommes et les femmes peut-il encore baisser?», Économie et Statistique no 398-399, pp. 99-129.

Meurs D., Pailhe A., Ponthieux S., (2008), "How much does it cost to stay at home? Career interruptions and the gender wage gap in France", Document de travail INSEE F0802.

Mincer J. (1974), Schooling, Experience and Earnings, Columbia University Press, New York.

Mulligan C.B., Rubinstein Y. (2008), "Selection, Investment, and Women's relative wages over time", Quarterly Journal of Economics, no 3, pp. 1061-1110.

NAKache J.-P., Confais J. (2005), Approche pragmatique de la classification, Technip, $256 \mathrm{p}$.

Nortier F., Rass C. (2006), «Les carrières s'aplatissent», Dossier Retraités et retraite 2005-2025, Retraite complémentaire AGIRC-ARRCO, $1^{\text {er }}$ trim., 20 p.

Willis R.G. (1986), "Wage Determinants: a Survey and Reinterpretation of Human Capital Earning Functions", in Ashenfelter O. and Layard R. eds. Handbook of Labor Economics, North Holland. 